\title{
Photocatalyzed Dehydroxylative Amination of Phenols: A Ring-Expansion Approach for Medium-Sized Benzolactams
}

Ying Fu,* Ruijuan Li, Mingpeng Li, Yongyan Zhang, Yuxia Zhang and Tianyu Yang

Key Laboratory of Eco-functional Polymer Materials of the Ministry of Education, College of Chemistry and Chemical Engineering, Northwest Normal University, Lanzhou 730070, P. R. China.

E-mail:fu_yingmail@126.com

\section{Table of Contents:}

$\begin{array}{lr}\text { 1. General Experimental Information } & \text { S2 }\end{array}$

2. Details of experimental procedures

2.1 General synthetic procedure for the coupling reaction of hydroxyphenols and S3

$\mathrm{N}$-alkyl-4-piperidinones

2.2 Compound characterization data $\quad$ S3

$\begin{array}{lr}2.3 \text { Turn on / off experiments } & \text { S17 }\end{array}$

$\begin{array}{ll}2.4 \text { Radical capture experiment by butylated hydroxytoluene (BHT) } & \text { S17 }\end{array}$

$\begin{array}{ll}2.5 \text { EPR tests } & \text { S18 }\end{array}$

$\begin{array}{lr}\text { 2.6 Fluorescence quenching experiments } & \text { S20 }\end{array}$

$\begin{array}{lr}2.7 \text { Cyclic voltammetry }(\mathrm{CV}) \text { experiments } & \text { S21 }\end{array}$

$\begin{array}{lr}\text { 3. Copies of NMR spectra of compounds synthesized. } & \text { S22 }\end{array}$ 


\section{General Experimental Information}

Analytic methods. All reactions were carried out under air atmosphere. ${ }^{1} \mathrm{H}$ NMR and ${ }^{13} \mathrm{CNMR}$ were recorded on Varian Mercury $400 \mathrm{MHz}$ or Bruker $600 \mathrm{NMR}$ with $\mathrm{CDCl}_{3}$ as solvent. Chemical shifts of ${ }^{1} \mathrm{H}$ and ${ }^{13} \mathrm{C}$ NMR spectra are reported in parts per million (ppm) with TMS as an internal standard. Data are reported as follows: chemical shift, integration, multiplicity $(\mathrm{s}=$ singlet, $\mathrm{d}=$ doublet, $\mathrm{t}=$ triplet, $\mathrm{q}=$ quartet, quint $=$ quintet, $\mathrm{sx}=$ sextet, $\mathrm{sept}=$ septet, $\mathrm{m}=$ multiplet and $\mathrm{br}=$ broad), and coupling constant $(J)$ are in Hz. High resolution mass spectra (HRMS) were performed using a 6520B Q-TOF high-resolution mass spectrometer. Infrared spectroscopy were acquired using a DIGLAB FTS-300 Fourier transform infrared spectrometer. Column chromatography was performed on silica gel 300-400 mesh. Analytical thin layer chromatography (TLC) was performed on pre-coated, glass-backed silica gel plates. All reagents were purchased from commercial sources and were used as thus.

The photoreactors used were custom designed and built in-house as shown in Figure S1. Beneath each hole, there is a $5 \times 1 \mathrm{~W}$ white lamp beam, purchased from Shenzhen Dezhigaoxing Co.,Ltd. Each side in this instrument, there is a fan to ventilate the room and keep the temperature inside around room temperature. The borosilicate glass Schlenk-tube used in these reactions were purchased from Beijing Synware Glass Co. Ltd. The distance from the light source to the irradiation vessel is $1.0 \mathrm{~cm}$.

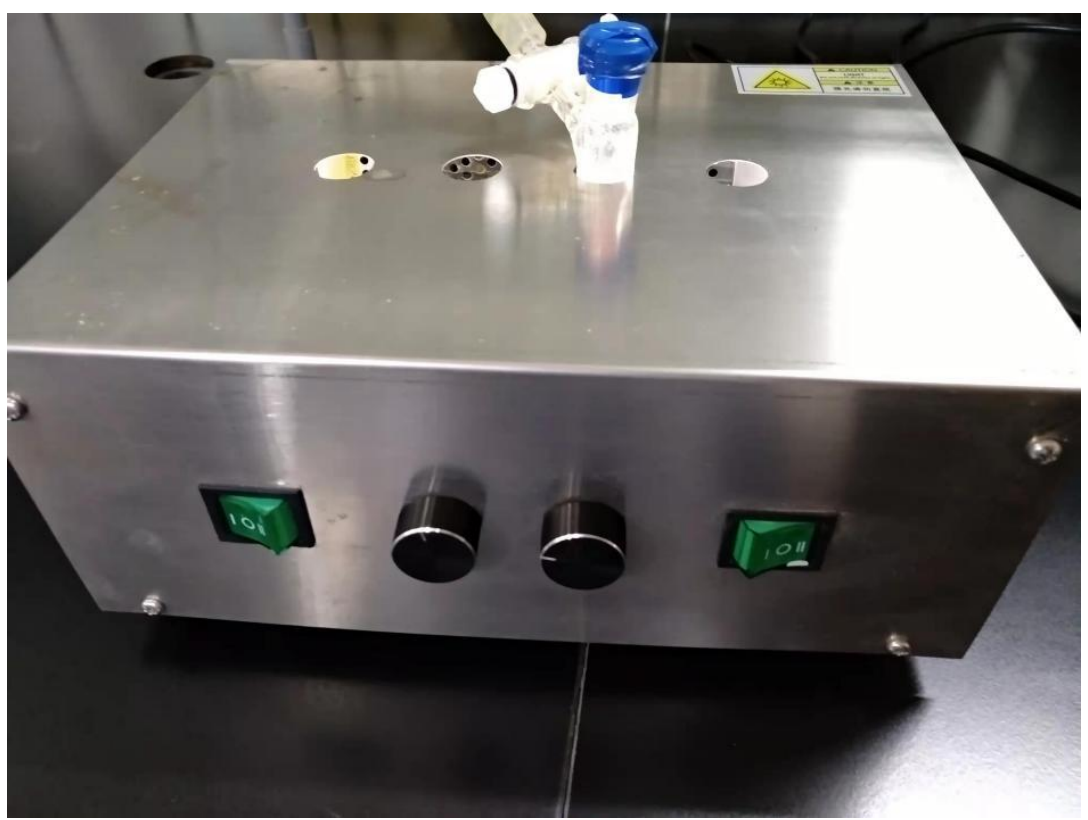

Figure S1. Photo of the lamp and the reaction 


\section{Details of experimental procedures}

\subsection{General procedure for the coupling reaction of hydroquinone and piperidinone}

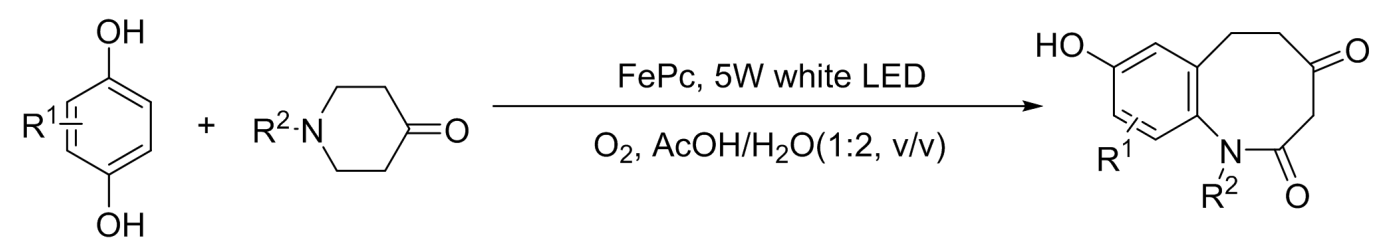

A $10 \mathrm{~mL}$ Schlenk-tube containing a stirring bar, phenols $(0.5 \mathrm{mmol})$, iron (II) phthalocyanine (11.4 $\mathrm{mg}, 2 \mathrm{mmol} \%$ ) was evacuated and purged with oxygen air for three times. N-alkylpiperidinones $(0.75 \mathrm{mmol})$, acetic acid $(1.0 \mathrm{~mL})$ and water $(2.0 \mathrm{~mL})$ were then added. After stirred and irradiated with white LED ( $5 \mathrm{~W}$ ) for 24 hours at room temperature, the reaction mixtures were quenched with aqueous $\mathrm{NaHCO}_{3}$ solution which was then dissolved in ethyl acetate $(10 \mathrm{~mL})$ and washed successively with water $(2 \times 10 \mathrm{~mL})$ and then brine $(10 \mathrm{~mL})$. The aqueous phase was further extracted from ethyl acetate $(10 \mathrm{~mL})$ and washed as previously. The organic phase was combined, dried over $\mathrm{Na}_{2} \mathrm{SO}_{4}$ and concentrated. Purification by silica gels column chromatography gave desired products.

\subsection{Compound characterization data}

\section{8-Hydroxy-1-methyl-5,6-dihydrobenzo[b]azocine-2,4(1H,3H)-dione (3a)}

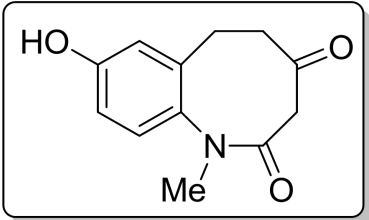

Yellowish oil. The reaction of hydroquinone $(55 \mathrm{mg}, 0.5 \mathrm{mmol}), 1$-methylpiperidin-4-one (86 $\mathrm{mg}, 0.75 \mathrm{mmol}$ ) and iron (II) phthalocyanine (11.4 mg, $2 \mathrm{mmol} \%$ ), affords $3 \mathrm{a}$ in $68 \%$ (74.5 mg) yield.

${ }^{1} \mathbf{H}$ NMR $\left(600 \mathrm{MHz}, \mathrm{CDCl}_{3}\right) \delta(\mathrm{ppm}): 7.23(\mathrm{dd}, J=8.9,2.8 \mathrm{~Hz}, 1 \mathrm{H}), 7.08(\mathrm{~d}, J=2.7 \mathrm{~Hz}, 1 \mathrm{H})$, $6.99(\mathrm{dd}, J=8.8,2.3 \mathrm{~Hz}, 1 \mathrm{H}), 3.22(\mathrm{td}, J=9.3,8.7,4.9 \mathrm{~Hz}, 1 \mathrm{H}), 3.08(\mathrm{~d}, J=10.8 \mathrm{~Hz}, 1 \mathrm{H}), 2.85-$ $2.79(\mathrm{~m}, 1 \mathrm{H}), 2.70(\mathrm{q}, J=8.3 \mathrm{~Hz}, 1 \mathrm{H}), 2.50(\mathrm{~s}, 3 \mathrm{H}), 2.38$ (ddd, $J=13.6,8.4,4.9 \mathrm{~Hz}, 1 \mathrm{H}), 2.27-$ $2.21(\mathrm{~m}, 1 \mathrm{H})$.

${ }^{13} \mathbf{C}\left\{{ }^{1} \mathbf{H}\right\}$ NMR (151 MHz, $\left.\mathrm{CDCl}_{3}\right) \delta(\mathrm{ppm}): 202.1,166.3,152.0,128.2,120.2,114.1,108.0$, $95.5,65.0,55.7,41.9,36.5$.

HRMS (ESI) $m / z$ : Calcd for $\mathrm{C}_{12} \mathrm{H}_{13} \mathrm{NO}_{3}[\mathrm{M}+\mathrm{H}]^{+} 220.0968$; Found 220.0966 . 


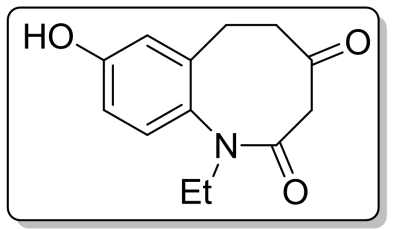

Yellowish oil. The reaction of hydroquinone $(55 \mathrm{mg}, 0.5 \mathrm{mmol})$, 1-ethylpiperidin-4-one (95.2 $\mathrm{mg}, 0.75 \mathrm{mmol})$ and iron (II) phthalocyanine (11.4 mg, 2 mmol\%), affords $3 \mathbf{b}$ in $63 \%$ (73.4 mg) yield.

${ }^{1}$ H NMR $\left(400 \mathrm{MHz}, \mathrm{CDCl}_{3}\right) \delta(\mathrm{ppm}): 7.23(\mathrm{dd}, J=8.8,2.7 \mathrm{~Hz}, 1 \mathrm{H}), 7.08(\mathrm{~d}, J=2.8 \mathrm{~Hz}, 1 \mathrm{H})$, $6.99(\mathrm{~d}, J=8.9 \mathrm{~Hz}, 1 \mathrm{H}), 3.26(\mathrm{td}, J=8.6,4.8 \mathrm{~Hz}, 1 \mathrm{H}), 3.14(\mathrm{~d}, J=10.9 \mathrm{~Hz}, 1 \mathrm{H}), 2.76(\mathrm{~d}, J=10.8$ $\mathrm{Hz}, 1 \mathrm{H}), 2.70-2.63(\mathrm{~m}, 3 \mathrm{H}), 2.41-2.31(\mathrm{~m}, 1 \mathrm{H}), 2.30-2.16(\mathrm{~m}, 1 \mathrm{H}), 1.17(\mathrm{t}, J=7.3 \mathrm{~Hz}, 3 \mathrm{H})$.

${ }^{13} \mathbf{C}\left\{{ }^{1} \mathbf{H}\right\}$ NMR (151 MHz, $\left.\mathrm{CDCl}_{3}\right) \delta(\mathrm{ppm}): 202.1,166.5,151.5,127.9,120.3,114.2,108.1$, $95.2,63.1,53.4,49.9,35.9,13.4$.

HRMS (ESI) $m / z$ : Calcd for $\mathrm{C}_{13} \mathrm{H}_{15} \mathrm{NO}_{3}[\mathrm{M}+\mathrm{H}]^{+}$234.1125; Found 234.1121.

8-Hydroxy-1-propyl-5,6-dihydrobenzo[b]azocine-2,4(1H,3H)-dione (3c)<smiles>O=C1CCc2cc(O)ccc2N(P(Br)C2CC2)C(=O)C1</smiles>

Yellowish oil. The reaction of hydroquinone (110 mg, $1.0 \mathrm{mmol}), 1$-propylpiperidin-4-one (212 mg, $1.5 \mathrm{mmol})$ and iron (II) phthalocyanine (23 mg, $2 \mathrm{mmol} \%)$, affords $3 \mathrm{c}$ in $64 \%$ (158 $\mathrm{mg})$ yield.

${ }^{1} \mathbf{H}$ NMR $\left(600 \mathrm{MHz} \mathrm{CDCl}_{3}\right) \delta(\mathrm{ppm}): 7.23(\mathrm{dd}, J=8.9,2.8 \mathrm{~Hz}, 1 \mathrm{H}), 7.08(\mathrm{~d}, J=2.7 \mathrm{~Hz}, 1 \mathrm{H})$, $6.97(\mathrm{~d}, J=8.9 \mathrm{~Hz}, 1 \mathrm{H}), 3.23(\mathrm{ddd}, J=9.4,7.8,4.8 \mathrm{~Hz}, 1 \mathrm{H}), 3.12(\mathrm{~d}, J=10.9 \mathrm{~Hz}, 1 \mathrm{H}), 2.76(\mathrm{~d}, J$ $=10.9 \mathrm{~Hz}, 1 \mathrm{H}), 2.64(\mathrm{q}, J=8.4 \mathrm{~Hz}, 2 \mathrm{H}), 2.53(\mathrm{td}, J=7.2,1.8 \mathrm{~Hz}, 2 \mathrm{H}), 2.34(\mathrm{ddd}, J=13.5,8.4$, $4.8 \mathrm{~Hz}, 1 \mathrm{H}), 2.22(\mathrm{ddd}, J=13.9,7.5,1.1 \mathrm{~Hz}, 1 \mathrm{H}), 1.61-1.53(\mathrm{~m}, 2 \mathrm{H}), 0.92(\mathrm{t}, J=7.4 \mathrm{~Hz}, 3 \mathrm{H})$.

${ }^{13} \mathbf{C}\left\{{ }^{1} \mathbf{H}\right\}$ NMR (151 MHz, $\left.\mathrm{CDCl}_{3}\right) \delta(\mathrm{ppm}):$ 202.5, 166.4, 151.7, 128.1, 120.3, 114.1, 108.1, $95.3,63.4,58.1,53.9,35.9,21.7,11.9$

HRMS (ESI) $m / z$ : Calcd for $\mathrm{C}_{14} \mathrm{H}_{17} \mathrm{NO}_{3}[\mathrm{M}+\mathrm{H}]^{+}$248.1281; Found 248.1276.

1-Butyl-8-hydroxy-5,6-dihydrobenzo[b]azocine-2,4(1H,3H)-dione (3d) 


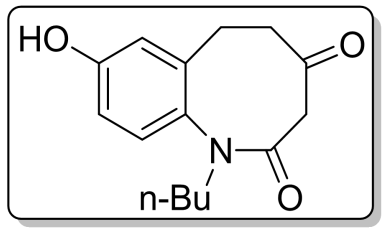

Yellowish oil. The reaction of hydroquinone (55 $\mathrm{mg}, 0.5 \mathrm{mmol}), 1$-butylpiperidin-4-one (116.5 mg, $0.75 \mathrm{mmol}$ ) and iron (II) phthalocyanine (11.4 mg, $2 \mathrm{mmol} \%$ ), affords $3 \mathbf{d}$ in $61 \%$ (79.6 mg) yield.

${ }^{1} \mathbf{H}$ NMR $\left(600 \mathrm{MHz}, \mathrm{CDCl}_{3}\right) \delta(\mathrm{ppm}): 7.23(\mathrm{dd}, J=8.9,2.8 \mathrm{~Hz}, 1 \mathrm{H}), 7.08(\mathrm{~d}, J=2.8 \mathrm{~Hz}, 1 \mathrm{H})$, $6.97(\mathrm{~d}, J=8.9 \mathrm{~Hz}, 1 \mathrm{H}), 3.28-3.22(\mathrm{~m}, 1 \mathrm{H}), 3.14(\mathrm{~d}, J=11.0 \mathrm{~Hz}, 1 \mathrm{H}), 2.78(\mathrm{~d}, J=11.0 \mathrm{~Hz}, 1 \mathrm{H})$, $2.67(\mathrm{q}, J=8.5 \mathrm{~Hz}, 1 \mathrm{H}), 2.59(\mathrm{td}, J=7.4,4.3 \mathrm{~Hz}, 2 \mathrm{H}), 2.34(\mathrm{ddd}, J=13.5,8.4,4.9 \mathrm{~Hz}, 1 \mathrm{H}), 2.21$ $(\mathrm{dt}, J=14.3,7.4 \mathrm{~Hz}, 1 \mathrm{H}), 1.59-1.46(\mathrm{~m}, 2 \mathrm{H}), 1.34(\mathrm{q}, J=7.4 \mathrm{~Hz}, 2 \mathrm{H}), 0.90(\mathrm{t}, J=7.4 \mathrm{~Hz}, 3 \mathrm{H})$

${ }^{13} \mathbf{C}\left\{{ }^{1} \mathbf{H}\right\}$ NMR (151 MHz,CDCl 3$) \delta(p p m): ~ 202.4,166.4,151.9,128.3,120.2,114.1,108.1$, $95.1,63.3,55.9,53.9,35.9,30.4,20.7,13.9$.

HRMS (ESI) $m / z$ : Calcd for $\mathrm{C}_{15} \mathrm{H}_{19} \mathrm{NO}_{3}[\mathrm{M}+\mathrm{H}]^{+}$262.1438; Found 262.1434.

8-Hydroxy-1-(3-phenylpropyl)-5,6-dihydrobenzo[b]azocine-2,4(1H,3H)-dione (3e)<smiles>CCN1C(=O)CC(=O)CCc2cc(O)ccc21</smiles>

The reaction of hydroquinone (55 mg, $0.5 \mathrm{mmol}), 1-(3$-phenylpropyl)piperidin-4-one (162.8 $\mathrm{mg}, 0.75 \mathrm{mmol}$ ) and iron (II) phthalocyanine (11.4 mg, $2 \mathrm{mmol} \%$ ), affords $3 \mathrm{e}$ in $52 \%(84.1 \mathrm{mg})$ yield.

${ }^{1}$ H NMR $\left(400 \mathrm{MHz}, \mathrm{CDCl}_{3}\right) \delta(\mathrm{ppm}): 7.28-7.14(\mathrm{~m}, 6 \mathrm{H}), 7.07(\mathrm{~d}, J=2.8 \mathrm{~Hz}, 1 \mathrm{H}), 6.96(\mathrm{~d}, J$ $=8.8 \mathrm{~Hz}, 1 \mathrm{H}), 3.21(\mathrm{td}, J=8.4,4.7 \mathrm{~Hz}, 1 \mathrm{H}), 3.10(\mathrm{~d}, J=10.9 \mathrm{~Hz}, 1 \mathrm{H}), 2.75(\mathrm{~d}, J=10.7 \mathrm{~Hz}, 1 \mathrm{H})$, 2.66-2.54 (m, 5H), 2.35-2.30 (m, 1H), $2.20(\mathrm{dt}, J=14.3,7.5 \mathrm{~Hz}, 1 \mathrm{H}), 1.88(\mathrm{p}, J=7.7 \mathrm{~Hz}, 2 \mathrm{H})$.

${ }^{13} \mathbf{C}\left\{{ }^{1} \mathbf{H}\right\}$ NMR (151 MHz,CDCl 3$) \delta(p p m): ~ 202.6,166.5,151.8,141.7,128.3$ (3C), 125.9, $120.2,114.2,108.0,95.2,63.4,55.5,53.8,35.9,33.7,30.0$.

HRMS (ESI) $m / z$ : Calcd for $\mathrm{C}_{20} \mathrm{H}_{21} \mathrm{NO}_{3}[\mathrm{M}+\mathrm{H}]^{+}$324.1594; Found 324.1592. 


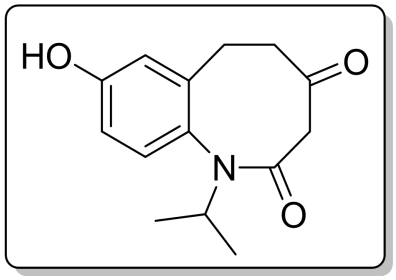

Yellowish oil. The reaction of hydroquinone (55 mg, $0.5 \mathrm{mmol}$ ), 1-isopropylpiperidin-4-one (105.8 mg, $0.75 \mathrm{mmol}$ ) and iron (II) phthalocyanine (11.4 mg, 2 mmol\%), affords $3 \mathbf{f}$ in $54 \%$ (66.7 $\mathrm{mg}$ ) yield.

${ }^{1} \mathrm{H}$ NMR $\left(600 \mathrm{MHz}, \mathrm{CDCl}_{3}\right) \delta(\mathrm{ppm}): 7.23(\mathrm{dd}, J=8.9,2.8 \mathrm{~Hz}, 1 \mathrm{H}), 7.10(\mathrm{~d}, J=2.7 \mathrm{~Hz}, 1 \mathrm{H})$, $6.97(\mathrm{~d}, J=8.9 \mathrm{~Hz}, 1 \mathrm{H}), 3.30-3.24(\mathrm{~m}, 1 \mathrm{H}), 3.18(\mathrm{~d}, J=10.8 \mathrm{~Hz}, 1 \mathrm{H}), 2.80(\mathrm{~d}, J=10.9 \mathrm{~Hz}, 1 \mathrm{H})$, $2.68(\mathrm{td}, J=8.9,7.3 \mathrm{~Hz}, 1 \mathrm{H}), 2.58(\mathrm{p}, J=6.3 \mathrm{~Hz}, 1 \mathrm{H}), 2.34(\mathrm{ddd}, J=13.6,8.6,4.9 \mathrm{~Hz}, 1 \mathrm{H}), 2.24$ $-2.16(\mathrm{~m}, 1 \mathrm{H}), 1.17(\mathrm{~d}, J=6.3 \mathrm{~Hz}, 3 \mathrm{H}), 1.14(\mathrm{~d}, J=6.3 \mathrm{~Hz}, 3 \mathrm{H})$.

${ }^{13} \mathbf{C}\left\{{ }^{1} \mathbf{H}\right\}$ NMR (151 MHz, $\left.\mathrm{CDCl}_{3}\right) \delta(\mathrm{ppm}): 202.5,166.4,151.7,128.3,120.2,114.1,108.2$, $95.1,61.5,55.0,51.6,35.8,21.3,21.2$.

HRMS (ESI) $m / z$ : Calcd for $\mathrm{C}_{14} \mathrm{H}_{17} \mathrm{NO}_{3}[\mathrm{M}+\mathrm{H}]^{+}$248.1281; Found 248.1276.

1-Cyclopropyl-8-hydroxy-5,6-dihydrobenzo $[b]$ azocine-2,4(1H,3H)-dione (3h)

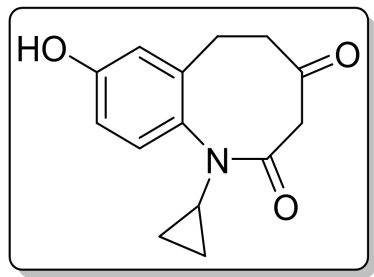

Yellowish oil. The reaction of hydroquinone (55 $\mathrm{mg}, 0.5 \mathrm{mmol}$ ), 1-cyclopropylpiperidin-4-one $(104.3 \mathrm{mg}, 0.75 \mathrm{mmol})$ and iron (II) phthalocyanine $(11.4 \mathrm{mg}, 2$ mmol\%), affords $3 \mathbf{h}$ in $55 \%(67.7 \mathrm{mg})$ yield.

${ }^{1} \mathbf{H}$ NMR $\left(600 \mathrm{MHz}, \mathrm{CDCl}_{3}\right) \delta(\mathrm{ppm}): 7.20(\mathrm{dd}, J=8.9,2.8 \mathrm{~Hz}, 1 \mathrm{H}), 7.06(\mathrm{~d}, J=2.8 \mathrm{~Hz}, 1 \mathrm{H})$, $6.99(\mathrm{~d}, J=8.8 \mathrm{~Hz}, 1 \mathrm{H}), 3.26(\mathrm{td}, J=8.7,5.1 \mathrm{~Hz}, 1 \mathrm{H}), 3.15(\mathrm{~d}, J=11.1 \mathrm{~Hz}, 1 \mathrm{H}), 2.96(\mathrm{~d}, J=11.0$ $\mathrm{Hz}, 1 \mathrm{H}), 2.85(\mathrm{q}, J=8.4 \mathrm{~Hz}, 1 \mathrm{H}), 2.34(\mathrm{ddd}, J=11.5,8.5,4.2 \mathrm{~Hz}, 1 \mathrm{H}), 2.22-2.12(\mathrm{~m}, 1 \mathrm{H}), 1.84$ (dt, $J=7.1,3.1 \mathrm{~Hz}, 1 \mathrm{H}), 0.63-0.44(\mathrm{~m}, 4 \mathrm{H})$.

${ }^{13} \mathbf{C}\left\{{ }^{1} \mathbf{H}\right\}$ NMR $\left(151 \mathrm{MHz}, \mathrm{CDCl}_{3}\right) \delta$ (ppm): 206.3, 166.6, 151.0, 127.6, 120.5, 114.2, 108.0, $95.9,63.5,53.7,36.2,35.5,5.9,5.8$.

HRMS (ESI) $m / z$ : Calcd for $\mathrm{C}_{14} \mathrm{H}_{15} \mathrm{NO}_{3}[\mathrm{M}+\mathrm{H}]^{+}$246.1125; Found 246.1120 . 


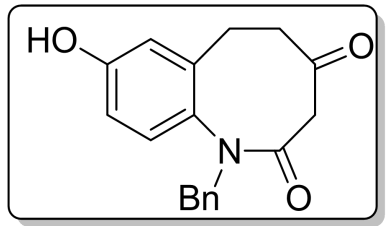

Yellowish oil. The reaction of hydroquinone $(55 \mathrm{mg}, 0.5 \mathrm{mmol})$, 1-benzylpiperidin-4-one (141.8 mg, $0.75 \mathrm{mmol}$ ) and iron (II) phthalocyanine (11.4 mg, 2 mmol\%), affords $3 \mathbf{i}$ in $42 \%$ (61.9 $\mathrm{mg}$ ) yield.

${ }^{1} \mathbf{H}$ NMR $\left(600 \mathrm{MHz}, \mathrm{CDCl}_{3}\right) \delta(\mathrm{ppm}): 7.35(\mathrm{~d}, J=7.2 \mathrm{~Hz}, 2 \mathrm{H}), 7.30(\mathrm{t}, J=7.2 \mathrm{~Hz}, 2 \mathrm{H}), 7.24$ $(\mathrm{t}, J=7.8 \mathrm{~Hz}, 1 \mathrm{H}), 7.18(\mathrm{dd}, J=9.0,2.8 \mathrm{~Hz}, 1 \mathrm{H}), 7.03(\mathrm{~d}, J=2.8 \mathrm{~Hz}, 1 \mathrm{H}), 6.96(\mathrm{~d}, J=8.9 \mathrm{~Hz}$, $1 \mathrm{H}), 3.80(\mathrm{~d}, J=12.8 \mathrm{~Hz}, 1 \mathrm{H}), 3.70(\mathrm{~d}, J=12.8 \mathrm{~Hz}, 1 \mathrm{H}), 3.21-3.14(\mathrm{~m}, 1 \mathrm{H}), 3.01(\mathrm{~d}, J=10.9$ $\mathrm{Hz}, 1 \mathrm{H}), 2.76(\mathrm{~d}, J=10.9 \mathrm{~Hz}, 1 \mathrm{H}), 2.69(\mathrm{dt}, J=9.4,7.9 \mathrm{~Hz}, 1 \mathrm{H}), 2.39-2.29(\mathrm{~m}, 1 \mathrm{H}), 2.22(\mathrm{dt}, J$ $=13.9,7.6 \mathrm{~Hz}, 1 \mathrm{H})$.

${ }^{13} \mathbf{C}\left\{{ }^{1} \mathbf{H}\right\}$ NMR $\left(151 \mathrm{MHz}, \mathrm{CDCl}_{3}\right) \delta(\mathrm{ppm}): 202.7,166.5,151.2,137.8,129.0,128.3,127.8$, $127.3,120.2,114.1,108.0,95.4,63.1,59.8,53.7,36.0$.

HRMS (ESI) $m / z$ : Calcd for $\mathrm{C}_{19} \mathrm{H}_{17} \mathrm{NO}_{3}[\mathrm{M}+\mathrm{H}]^{+}$296.1281; Found 296.1274 .

\section{1-Allyl-8-hydroxy-5,6-dihydrobenzo[b]azocine-2,4(1H,3H)-dione $(3 \mathrm{j})$}<smiles>C=CC1CCC(=O)CC(=O)CN1C</smiles>

Brownish oil. The reaction of hydroquinone ( $55 \mathrm{mg}, 0.5 \mathrm{mmol}$ ), 1-allylpiperidin-4-one (104.4 $\mathrm{mg}, 0.75 \mathrm{mmol}$ ) and iron (II) phthalocyanine (11.4 mg, 2 mmol\%), affords $3 \mathbf{j}$ in $44 \%$ (53.9 mg) yield.

${ }^{1} \mathbf{H}$ NMR $\left(600 \mathrm{MHz}, \mathrm{CDCl}_{3}\right) \delta(\mathrm{ppm}): 7.21(\mathrm{dd}, J=8.9,2.8 \mathrm{~Hz}, 1 \mathrm{H}), 7.06(\mathrm{~d}, J=2.8 \mathrm{~Hz}, 1 \mathrm{H})$, $6.98(\mathrm{~d}, J=8.9 \mathrm{~Hz}, 1 \mathrm{H}), 6.00-5.89(\mathrm{~m}, 1 \mathrm{H}), 5.26(\mathrm{dq}, J=17.1,1.5 \mathrm{~Hz}, 1 \mathrm{H}), 5.18(\mathrm{dd}, J=9.8$, $1.3 \mathrm{~Hz}, 1 \mathrm{H}), 3.36-3.28(\mathrm{~m}, 2 \mathrm{H}), 3.28-3.22(\mathrm{~m}, 1 \mathrm{H}), 3.14(\mathrm{~d}, J=11.3 \mathrm{~Hz}, 1 \mathrm{H}), 2.86(\mathrm{~d}, J=11.3$ $\mathrm{Hz}, 1 \mathrm{H}), 2.78(\mathrm{ddd}, J=9.7,8.4,6.9 \mathrm{~Hz}, 1 \mathrm{H}), 2.36(\mathrm{ddd}, J=13.9,8.4,5.5 \mathrm{~Hz}, 1 \mathrm{H}), 2.22(\mathrm{dtd}, J=$ $14.0,7.7,7.3,1.2 \mathrm{~Hz}, 1 \mathrm{H})$. 
${ }^{13} \mathbf{C}\left\{{ }^{1} \mathbf{H}\right\}$ NMR (151 MHz, $\left.\mathrm{CDCl}_{3}\right) \delta(\mathrm{ppm}): 201.8,166.3,151.6,133.8,127.9,120.2,118.9$, $114.2,108.0,94.9,62.4,58.5,53.2,35.8$.

HRMS (ESI) $m / z$ : Calcd for $\mathrm{C}_{14} \mathrm{H}_{15} \mathrm{NO}_{3}[\mathrm{M}+\mathrm{H}]^{+} 246.1125$; Found 246.1121.

\section{8-Hydroxy-1-(prop-2-yn-1-yl)-5,6-dihydrobenzo[b]azocine-2, 4(1H,3H)-dione (3k)}

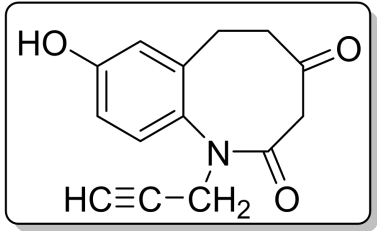

Yellowish oil. The reaction of hydroquinone (55 $\mathrm{mg}, 0.5 \mathrm{mmol})$, 1-(prop-2-yn-1-yl)piperidin-4-one (102.8 mg, $0.75 \mathrm{mmol})$ and iron (II) phthalocyanine $(11.4 \mathrm{mg}, 2$ mmol\%), affords $3 \mathbf{k}$ in $52 \%(63.3 \mathrm{mg})$ yield.

${ }^{1} \mathbf{H}$ NMR $\left(400 \mathrm{MHz}, \mathrm{CDCl}_{3}\right) \delta(\mathrm{ppm}): 7.22(\mathrm{ddd}, J=8.9,2.8,0.8 \mathrm{~Hz}, 1 \mathrm{H}), 7.08(\mathrm{dt}, J=2.8$, $0.7 \mathrm{~Hz}, 1 \mathrm{H}), 7.01(\mathrm{dt}, J=8.9,0.7 \mathrm{~Hz}, 1 \mathrm{H}), 6.73(\mathrm{~d}, J=0.8 \mathrm{~Hz}, 1 \mathrm{H}), 3.56(\mathrm{t}, J=2.0 \mathrm{~Hz}, 2 \mathrm{H}), 3.46$ $(\mathrm{dd}, J=2.5,0.8 \mathrm{~Hz}, 1 \mathrm{H}), 3.19(\mathrm{td}, J=8.5,5.1 \mathrm{~Hz}, 1 \mathrm{H}), 3.06(\mathrm{~d}, J=10.5 \mathrm{~Hz}, 1 \mathrm{H}), 2.98(\mathrm{~d}, J=$ $10.5 \mathrm{~Hz}, 1 \mathrm{H}), 2.42-2.35(\mathrm{~m}, 1 \mathrm{H}), 2.31-2.28(\mathrm{~m}, 1 \mathrm{H}), 2.26-2.19(\mathrm{~m}, 1 \mathrm{H})$.

${ }^{13} \mathbf{C}\left\{{ }^{1} \mathbf{H}\right\}$ NMR (151 MHz,CDCl 3$) \delta(\mathrm{ppm}): 201.8,166.5,151.2,127.6,120.4,116.2,114.2$, $108.1,95.5,77.9,73.5,51.8,41.0,36.2$.

HRMS (ESI) $m / z$ : Calcd for $\mathrm{C}_{14} \mathrm{H}_{13} \mathrm{NO}_{3}[\mathrm{M}+\mathrm{H}]^{+}$244.0968; Found 244.0963 .

\section{Ethyl 2-(8-hydroxy-2,4-dioxo-3,4,5,6-tetrahydrobenzo[b]azocin-1(2H)-yl)acetate (3I)}<smiles>CCOC(=O)CN1C(=O)CC(=O)CCc2cc(O)ccc21</smiles>

Yellowish oil. The reaction of hydroquinone (55 mg, $0.5 \mathrm{mmol}$ ), ethyl 2-(4-oxopiperidin-1-yl) acetate $(138.8 \mathrm{mg}, 0.75 \mathrm{mmol})$ and iron (II) phthalocyanine $(11.4 \mathrm{mg}, 2 \mathrm{mmol} \%)$, affords $3 \mathrm{l}$ in $54 \%(78.6 \mathrm{mg})$ yield.

${ }^{1} \mathbf{H}$ NMR $\left(400 \mathrm{MHz}, \mathrm{CDCl}_{3}\right) \delta(\mathrm{ppm}): 7.25(\mathrm{ddd}, J=8.8,2.8,1.6 \mathrm{~Hz}, 1 \mathrm{H}), 7.10(\mathrm{dd}, J=2.8$, $1.6 \mathrm{~Hz}, 1 \mathrm{H}), 6.97(\mathrm{dd}, J=8.9,1.3 \mathrm{~Hz}, 1 \mathrm{H}), 4.32-4.02(\mathrm{~m}, 2 \mathrm{H}), 3.49(\mathrm{dd}, J=8.9,1.3 \mathrm{~Hz}, 1 \mathrm{H})$, $3.38(\mathrm{~d}, J=1.3 \mathrm{~Hz}, 1 \mathrm{H}), 3.24$ (d, $J=10.8 \mathrm{~Hz}, 1 \mathrm{H}), 3.04-2.77$ (m, 2H), 2.53 (s, 1H), $2.44-2.27$ (m, 1H), $2.22(\mathrm{dt}, J=13.9,7.1 \mathrm{~Hz}, 1 \mathrm{H}), 1.27(\mathrm{td}, J=7.0,1.3 \mathrm{~Hz}, 3 \mathrm{H})$. 
${ }^{13} \mathbf{C}\left\{{ }^{1} \mathbf{H}\right\}$ NMR (151 MHz, $\left.\mathrm{CDCl}_{3}\right) \delta(\mathrm{ppm}):$ 202.3, 170.3, 166.4, 151.7, 128.0, 120.1, 114.1, $107.9,95.4,62.8,55.9,53.5,41.0,36.0,14.1$.

HRMS (ESI) $m / z$ : Calcd for $\mathrm{C}_{15} \mathrm{H}_{17} \mathrm{NO}_{5}[\mathrm{M}+\mathrm{H}]^{+}$292.1179; Found 292.1176.

\section{8-Hydroxy-1,9-dimethyl-5,6-dihydrobenzo[b]azocine-2,4(1H,3H)-dione (4a)}

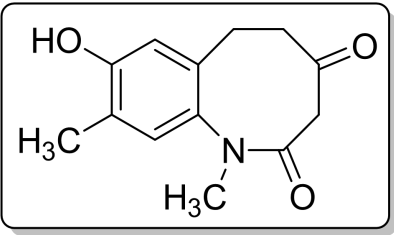

and

8-Hydroxy-1,7-dimethyl-5,6-dihydrobenzo $[b]$ azocine-2,4(1H,3H)-dione (4a')

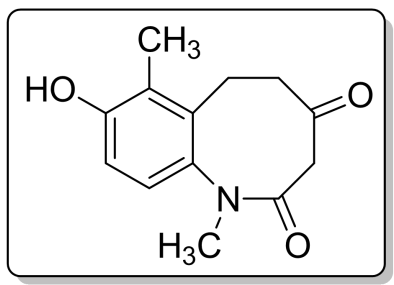

Yellowish oil. The reaction of 2-methylbenzene-1,4-diol (62 $\mathrm{mg}, 0.5 \mathrm{mmol})$, 1-methylpiperidin-4-one (86 mg, $0.75 \mathrm{mmol})$ and iron (II) phthalocyanine (11.4 mg, $2 \mathrm{mmol} \%$ ), affords $\mathbf{4 a}$ and $\mathbf{4 a}$ ' in $65 \mathrm{mg}$ (56\% yield) as an inseparable mixture in about 3:1 ratio.

${ }^{1} \mathbf{H}$ NMR $\left(600 \mathrm{MHz}, \mathrm{CDCl}_{3}\right) \delta(\mathrm{ppm}): 7.14(\mathrm{~d}, J=8.7 \mathrm{~Hz}, 0.35 \mathrm{H}), 7.04(\mathrm{dd}, J=2.7,1.1 \mathrm{~Hz}, 1 \mathrm{H})$, $6.88(\mathrm{~d}, J=2.8 \mathrm{~Hz}, 1 \mathrm{H}), 6.77(\mathrm{~d}, J=8.7 \mathrm{~Hz}, 0.35 \mathrm{H}), 3.20-3.15(\mathrm{~m}, 1.35 \mathrm{H}), 3.08-2.96(\mathrm{~m}, 1.35 \mathrm{H})$, $2.74-2.68(\mathrm{~m}, 1.35 \mathrm{H}), 2.65-2.61(\mathrm{~m}, 1.35 \mathrm{H}), 2.48(\mathrm{~s}, 3 \mathrm{H}), 2.45(\mathrm{~s}, 1.2 \mathrm{H}), 2.36-2.30(\mathrm{~m}, 1.35 \mathrm{H})$, $2.26-2.16(\mathrm{~m}, 1.35 \mathrm{H})$

${ }^{13} \mathbf{C}\left\{{ }^{1} \mathbf{H}\right\}$ NMR $\left(151 \mathrm{MHz}, \mathrm{CDCl}_{3}\right) \delta(\mathrm{ppm}): 202.9,202.8,166.6,165.6,151.6,149.1,128.5$, $126.2,124.6,123.4,119.5,118.6,114.7,110.3,106.7,104.9,95.5,95.1,65.4,65.2,55.9,55.8,41.9$, $41.8,36.7,36.6,36.5,17.9,14.4$

HRMS (ESI) $m / z$ : Calcd for $\mathrm{C}_{13} \mathrm{H}_{15} \mathrm{NO}_{3}[\mathrm{M}+\mathrm{H}]^{+}$234.1125; Found 234.1122. 


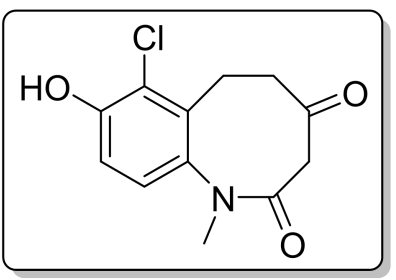

Brownish oil. The reaction of 2-chlorobenzene-1,4-diol (71.9 $\mathrm{mg}, 0.5 \mathrm{mmol})$, 1-methylpiperidin-4-one (86 mg, $0.75 \mathrm{mmol})$ and iron (II) phthalocyanine $(11.4 \mathrm{mg}, 2 \mathrm{mmol} \%)$, affords $\mathbf{4 b}$ in $35 \%$ (44 mg) yield.

${ }^{1}$ H NMR (400 MHz, $\left.\mathrm{CDCl}_{3}\right) \delta(\mathrm{ppm}): 7.33(\mathrm{~d}, J=8.9 \mathrm{~Hz}, 1 \mathrm{H}), 6.95(\mathrm{dd}, J=8.9,0.9 \mathrm{~Hz}, 1 \mathrm{H})$, $5.31(\mathrm{~s}, 1 \mathrm{H}), 3.26-3.15(\mathrm{~m}, 1 \mathrm{H}), 3.03(\mathrm{~d}, J=10.7 \mathrm{~Hz}, 1 \mathrm{H}), 2.71(\mathrm{~d}, J=10.7 \mathrm{~Hz}, 1 \mathrm{H}), 2.58(\mathrm{q}, J=$ $8.3 \mathrm{~Hz}, 1 \mathrm{H}), 2.45(\mathrm{~s}, 3 \mathrm{H}), 2.44-2.34(\mathrm{~m}, 1 \mathrm{H}), 2.28-2.16(\mathrm{~m}, 1 \mathrm{H})$.

${ }^{13} \mathbf{C}\left\{{ }^{1} \mathbf{H}\right\}$ NMR $\left(151 \mathrm{MHz}, \mathrm{CDCl}_{3}\right) \delta(\mathrm{ppm}): 199.2,166.7,147.1,126.6,116.9,114.1,112.6$, $96.5,65.4,55.7,41.7,36.7$.

HRMS (ESI) $m / z$ : Calcd for $\mathrm{C}_{13} \mathrm{H}_{15} \mathrm{NO}_{3}[\mathrm{M}+\mathrm{H}]^{+}$254.0506; Found 254.0502 .

\section{8-Hydroxy-1,9,10-trimethyl-5,6-dihydrobenzo $[b]$ azocine-2,4(1H,3H)-dione (4c)}

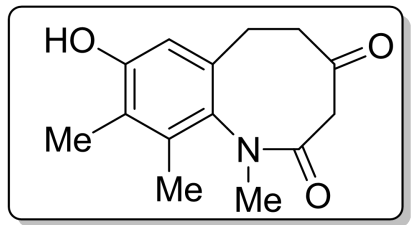

Yellow oil. The reaction of 2,3-dimethylbenzene-1,4-diol (69 $\mathrm{mg}, 0.5 \mathrm{mmol})$, 1-methylpiperidin-4-one (86 mg, $0.75 \mathrm{mmol})$ and iron (II) phthalocyanine $(11.4 \mathrm{mg}, 2 \mathrm{mmol} \%$ ), affords $4 \mathrm{c}$ in $64 \%(79.1 \mathrm{mg})$ yield.

${ }^{1} \mathbf{H}$ NMR $\left(600 \mathrm{MHz}^{\mathrm{CDCl}}{ }_{3}\right) \delta(\mathrm{ppm}): 6.83(\mathrm{~s}, 1 \mathrm{H}), 3.16(\mathrm{td}, J=8.6,4.2 \mathrm{~Hz}, 1 \mathrm{H}), 3.00(\mathrm{~d}, J=$ $10.6 \mathrm{~Hz}, 1 \mathrm{H}), 2.69$ (d, $J=10.6 \mathrm{~Hz}, 1 \mathrm{H}), 2.57$ (q, $J=8.6 \mathrm{~Hz}, 1 \mathrm{H}), 2.45$ (s, 3H), 2.33 (ddd, $J=13.8$, 8.3, 4.3 Hz, 2H), 2.25 (s, 3H), $2.23(\mathrm{~s}, 3 \mathrm{H})$.

${ }^{13} \mathbf{C}\left\{{ }^{1} \mathbf{H}\right\}$ NMR $\left(151 \mathrm{MHz}, \mathrm{CDCl}_{3}\right) \delta(\mathrm{ppm}): 202.7,165.9,150.1,137.3,122.7,116.5,103.9$, $95.0,65.4,56.0,41.9,36.7,13.2,11.3$.

HRMS (ESI) $m / z$ : Calcd for $\mathrm{C}_{14} \mathrm{H}_{17} \mathrm{NO}_{3}[\mathrm{M}+\mathrm{H}]^{+}$248.1281; Found 248.1279.

1-Ethyl-8-hydroxy-9,10-dimethyl-5,6-dihydrobenzo[b]azocine-2,4(1H,3H)-dione (4d) 
<smiles>CCN1C(=O)CC(=O)Cc2cc(O)c(C)c(C)c21</smiles>

Yellowish oil. The reaction of 2,3-dimethylbenzene-1,4-diol (69 $\mathrm{mg}, 0.5 \mathrm{mmol}$ ), 1-ethylpiperidin-4-one $(95.2 \mathrm{mg}, 0.75 \mathrm{mmol})$ and iron (II) phthalocyanine $(11.4 \mathrm{mg}, 2 \mathrm{mmol} \%)$, affords $4 \mathbf{d}$ in $69 \%(90.1 \mathrm{mg})$ yield.

${ }^{1}$ H NMR (600 MHz, $\left.\mathrm{CDCl}_{3}\right) \delta(\mathrm{ppm}): 6.87(\mathrm{~s}, 1 \mathrm{H}), 3.22-3.16(\mathrm{~m}, 1 \mathrm{H}), 3.08(\mathrm{~d}, J=10.7 \mathrm{~Hz}$, 1H), $2.70(\mathrm{~d}, J=10.8 \mathrm{~Hz}, 1 \mathrm{H}), 2.63-2.43(\mathrm{~m}, 2 \mathrm{H}), 2.34-2.28(\mathrm{~m}, 1 \mathrm{H}), 2.25(\mathrm{~s}, 3 \mathrm{H}), 2.21(\mathrm{~s}, 3 \mathrm{H})$, $1.57(\mathrm{~d}, J=7.6 \mathrm{~Hz}, 2 \mathrm{H}), 0.93(\mathrm{t}, J=7.4 \mathrm{~Hz}, 3 \mathrm{H})$.

${ }^{13} \mathbf{C}\left\{{ }^{1} \mathbf{H}\right\}$ NMR (151 MHz, $\left.\mathrm{CDCl}_{3}\right) \delta(\mathrm{ppm}):$ 202.7, 165.9, 149.7, 137.0, 122.8, 116.6, 103.9, $94.7,58.1,54.2,36.1,21.8,13.2,12.0,11.3$.

HRMS (ESI) $m / z$ : Calcd for $\mathrm{C}_{15} \mathrm{H}_{19} \mathrm{NO}_{3}[\mathrm{M}+\mathrm{H}]^{+}$262.1438; Found 262.1435.

8-Hydroxy-9,10-dimethyl-1-propyl-5,6-dihydrobenzo[b]azocine-2,4(1H,3H)-dione (4e)<smiles>CCCCN1C(=O)CC(=O)CCc2cc(O)c(C)c(C)c21</smiles>

Yellowish oil. The reaction of 2,3-dimethylbenzene-1,4-diol (69 $\mathrm{mg}, 0.5 \mathrm{mmol}$ ), 1-propylpiperidin-4-one (105.8 mg, $0.75 \mathrm{mmol})$ and iron (II) phthalocyanine (11.4 mg, $2 \mathrm{mmol} \%$ ), affords $4 \mathrm{e}$ in $65 \%(89.4 \mathrm{mg})$ yield.

${ }^{1}$ H NMR $\left(600 \mathrm{MHz}, \mathrm{CDCl}_{3}\right) \delta(\mathrm{ppm}): 6.89(\mathrm{~s}, 1 \mathrm{H}), 3.26-3.19(\mathrm{~m}, 1 \mathrm{H}), 3.11(\mathrm{~d}, J=10.8 \mathrm{~Hz}$, 1H), $2.72(\mathrm{~d}, J=10.8 \mathrm{~Hz}, 1 \mathrm{H}), 2.66-2.46(\mathrm{~m}, 3 \mathrm{H}), 2.36-2.30(\mathrm{~m}, 1 \mathrm{H}), 2.23(\mathrm{~s}, 3 \mathrm{H}), 2.23-2.18(\mathrm{~m}$, 1H), $2.17(\mathrm{~s}, 3 \mathrm{H}), 1.57$ (q, $J=7.6 \mathrm{~Hz}, 2 \mathrm{H}), 0.92(\mathrm{t}, J=7.4 \mathrm{~Hz}, 3 \mathrm{H})$.

${ }^{13} \mathbf{C}\left\{{ }^{1} \mathbf{H}\right\}$ NMR (151 MHz, $\left.\mathrm{CDCl}_{3}\right) \delta(\mathrm{ppm}): 202.9,165.9,150.1,137.6,122.7,116.4,103.8$, $94.5,63.5,58.1,54.2,36.0,21.7,13.3,12.0,11.3$.

HRMS (ESI) $m / z$ : Calcd for $\mathrm{C}_{16} \mathrm{H}_{21} \mathrm{NO}_{3}[\mathrm{M}+\mathrm{H}]^{+}$276.1594; Found 276.1591.

1-Butyl-8-hydroxy-9,10-dimethyl-5,6-dihydrobenzo[b]azocine-2,4(1H,3H)-dione (4f) 




Yellow oil. The reaction of 2,3-dimethylbenzene-1,4-diol (69 mg, $0.5 \mathrm{mmol}$ ), 1-butylpiperidin-4-one (116.5 mg, $0.75 \mathrm{mmol})$ and iron (II) phthalocyanine (11.4 mg, $2 \mathrm{mmol} \%$ ), affords $4 \mathbf{f}$ in $53 \%(76.6 \mathrm{mg})$ yield.

${ }^{1} \mathbf{H}$ NMR $\left(600 \mathrm{MHz}, \mathrm{CDCl}_{3}\right) \delta(\mathrm{ppm}): 6.89(\mathrm{~s}, 1 \mathrm{H}), 3.24-3.18(\mathrm{~m}, 1 \mathrm{H}), 3.10(\mathrm{~d}, J=10.8 \mathrm{~Hz}$, 1H), $2.72(\mathrm{~d}, J=10.8 \mathrm{~Hz}, 1 \mathrm{H}), 2.64-2.51(\mathrm{~m}, 3 \mathrm{H}), 2.31(\mathrm{ddd}, J=13.7,8.2,4.5 \mathrm{~Hz}, 1 \mathrm{H}), 2.24(\mathrm{~s}$, 3H), $2.21(\mathrm{ddd}, J=13.8,6.9,1.0 \mathrm{~Hz}, 1 \mathrm{H}), 2.18(\mathrm{~s}, 3 \mathrm{H}), 1.53(\mathrm{tt}, J=8.0,6.5 \mathrm{~Hz}, 2 \mathrm{H}), 1.39-1.31$ (m, 2H), $0.91(\mathrm{t}, J=7.4 \mathrm{~Hz}, 3 \mathrm{H})$.

${ }^{13} \mathbf{C}\left\{{ }^{1} \mathbf{H}\right\}$ NMR $\left(151 \mathrm{MHz}, \mathrm{CDCl}_{3}\right) \delta$ (ppm): 202.9, 165.8, 150.1, 137.6, 122.7, 116.4, 103.8, $94.5,63.6,56.0,54.2,36.0,30.6,20.7,13.96,13.3,11.3$.

HRMS (ESI) $m / z$ : Calcd for $\mathrm{C}_{17} \mathrm{H}_{23} \mathrm{NO}_{3}[\mathrm{M}+\mathrm{H}]^{+}$290.1751; Found 290.1747.

\section{8-Hydroxy-1,7,9-trimethyl-5,6-dihydrobenzo $[b]$ azocine-2,4(1H, 3H)-dione (4g)}<smiles>CCC1CC(=O)CCc2c1cc(C)c(O)c2C</smiles>

Yellowish oil. The reaction of 2,6-dimethylbenzene-1,4-diol (69 mg, $0.5 \mathrm{mmol}$ ), 1-methylpiperidin-4-one (86 mg, $0.75 \mathrm{mmol})$ and iron (II) phthalocyanine $(11.4 \mathrm{mg}, 2 \mathrm{mmol} \%$ ), affords $\mathbf{4 g}$ in $43 \%(53.1 \mathrm{mg})$ yield.

${ }^{1} \mathbf{H}$ NMR $\left(400 \mathrm{MHz} \mathrm{CDCl}_{3}\right) \delta(\mathrm{ppm}): 6.71(\mathrm{~s}, 1 \mathrm{H}), 3.16(\mathrm{dt}, J=8.7,4.3 \mathrm{~Hz}, 1 \mathrm{H}), 2.96(\mathrm{~d}, J=$ $10.6 \mathrm{~Hz}, 1 \mathrm{H}), 2.68(\mathrm{dd}, J=11.1,3.1 \mathrm{~Hz}, 1 \mathrm{H}), 2.59-2.53(\mathrm{~m}, 1 \mathrm{H}), 2.51(\mathrm{~s}, 3 \mathrm{H}), 2.45(\mathrm{~s}, 3 \mathrm{H}), 2.39$ $-2.34(\mathrm{~m}, 1 \mathrm{H}), 2.33(\mathrm{~s}, 3 \mathrm{H}), 2.22-2.12(\mathrm{~m}, 1 \mathrm{H})$

${ }^{13} \mathbf{C}\left\{{ }^{1} \mathbf{H}\right\}$ NMR (151 MHz, $\left.\mathrm{CDCl}_{3}\right) \delta(\mathrm{ppm}):$ 202.4, 166.6, 147.1, 137.0, 121.3, 116.5, 111.6, $95.2,65.4,55.8,41.7,36.6,17.8,10.1$

HRMS (ESI) $m / z$ : Calcd for $\mathrm{C}_{14} \mathrm{H}_{17} \mathrm{NO}_{3}[\mathrm{M}+\mathrm{H}]^{+}$248.1281; Found 248.1283. 


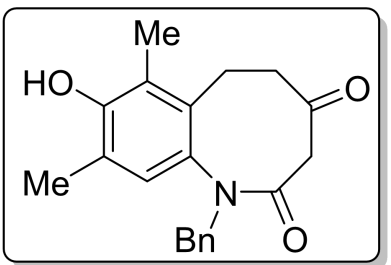

Yellowish oil. The reaction of 2,6-dimethylbenzene-1,4-diol (69 $\mathrm{mg}, 0.5 \mathrm{mmol})$, 1-benzylpiperidin-4-one (141 mg, $0.75 \mathrm{mmol})$ and iron (II) phthalocyanine (11.4 mg, $2 \mathrm{mmol} \%)$, affords $4 \mathrm{~h}$ in $41 \%(66 \mathrm{mg})$ yield.

${ }^{1} \mathbf{H}$ NMR $\left(400 \mathrm{MHz}, \mathrm{CDCl}_{3}\right) \delta 7.37(\mathrm{~d}, J=8.4 \mathrm{~Hz}, 2 \mathrm{H}), 7.31(\mathrm{ddd}, J=7.6,6.2,1.3 \mathrm{~Hz}, 2 \mathrm{H})$, $7.25-7.22(\mathrm{~m}, 2 \mathrm{H}), 6.71(\mathrm{~s}, 1 \mathrm{H}), 3.78(\mathrm{~d}, J=12.8 \mathrm{~Hz}, 1 \mathrm{H}), 3.69(\mathrm{~d}, J=12.8 \mathrm{~Hz}, 1 \mathrm{H}), 3.17(\mathrm{td}, J$ $=8.4,4.5 \mathrm{~Hz}, 1 \mathrm{H}), 2.98(\mathrm{~d}, J=10.7 \mathrm{~Hz}, 1 \mathrm{H}), 2.77-2.59(\mathrm{~m}, 2 \mathrm{H}), 2.49(\mathrm{~s}, 3 \mathrm{H}), 2.31(\mathrm{~s}, 3 \mathrm{H}), 2.23$ $-2.13(\mathrm{~m}, 2 \mathrm{H})$

${ }^{13} \mathbf{C}\left\{{ }^{1} \mathbf{H}\right\}$ NMR $\left(151 \mathrm{MHz}, \mathrm{CDCl}_{3}\right) \delta$ 206.6, 166.6, 147.1, 136.9, 136.4, 128.9, 128.3, 127.1, $121.2,116.5,111.6,94.5,59.9,53.8,36.1,29.7,17.9,10.1$.

HRMS (ESI) $m / z$ : Calcd for $\mathrm{C}_{20} \mathrm{H}_{22} \mathrm{NO}_{3}[\mathrm{M}+\mathrm{H}]^{+}$324.1594; Found 324.1590.

7,10-Dibromo-8-hydroxy-1-methyl-5,6-dihydrobenzo[b]azocine-2,4(1H,3H)-dione (4i)<smiles>CN1C(=O)CCCc2c(Br)c(O)cc(Br)c21</smiles>

Yellowish oil. The reaction of 2,5-dibromobenzene-1,4-diol (133.95 mg, $0.5 \mathrm{mmol}$ ), 1-methylpiperidin-4-one (86 mg, $0.75 \mathrm{mmol})$ and iron (II) phthalocyanine (11.4 mg, $2 \mathrm{mmol} \%$ ), affords $4 \mathbf{i}$ in $70 \%$ (131.2 $\mathrm{mg})$ yield.

${ }^{1} \mathbf{H}$ NMR $\left(600 \mathrm{MHz}, \mathrm{CDCl}_{3}\right) \delta(\mathrm{ppm}): 7.54(\mathrm{~s}, 1 \mathrm{H}), 3.20-3.12(\mathrm{~m}, 1 \mathrm{H}), 3.08(\mathrm{~d}, J=11.0 \mathrm{~Hz}$, 1H), $2.79(\mathrm{~d}, J=10.9 \mathrm{~Hz}, 1 \mathrm{H}), 2.65(\mathrm{dt}, J=9.2,7.8 \mathrm{~Hz}, 1 \mathrm{H}), 2.47(\mathrm{~s}, 3 \mathrm{H}), 2.38$ (ddd, $J=13.9,8.1$, $4.7 \mathrm{~Hz}, 1 \mathrm{H}), 2.33-2.25(\mathrm{~m}, 1 \mathrm{H})$

${ }^{13} \mathbf{C}\left\{{ }^{1} \mathbf{H}\right\}$ NMR $\left(151 \mathrm{MHz}, \mathrm{CDCl}_{3}\right) \delta(\mathrm{ppm}): 199.5,163.9,148.7,128.4,118.9,105.9,103.0$, $97.4,65.4,55.9,41.8,37.0$.

HRMS (ESI) $m / z$ : Calcd for $\mathrm{C}_{12} \mathrm{H}_{11} \mathrm{Br}_{2} \mathrm{NO}_{3}[\mathrm{M}+\mathrm{H}]^{+}$375.9178; Found 375.9175 . 


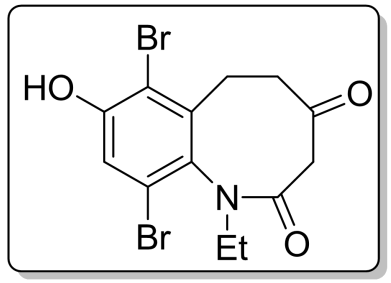

Yellowish oil. The reaction of 2,5-dibromobenzene-1,4-diol (133.95 $\mathrm{mg}, 0.5 \mathrm{mmol})$, 1-ethylpiperidin-4-one $(95.2 \mathrm{mg}, 0.75 \mathrm{mmol})$ and iron (II) phthalocyanine (11.4 mg, $2 \mathrm{mmol} \%$ ), affords $4 \mathbf{j}$ in $69 \%$ (134.8 $\mathrm{mg})$ yield.

${ }^{1}$ H NMR (600 MHz, DMSO-d $\left.d_{6}\right) \delta(\mathrm{ppm}): 10.53(\mathrm{~s}, 1 \mathrm{H}), 7.52(\mathrm{~s}, 1 \mathrm{H}), 3.05-2.92(\mathrm{~m}, 2 \mathrm{H})$, $2.61-2.53(\mathrm{~m}, 1 \mathrm{H}), 2.46-2.37(\mathrm{~m}, 2 \mathrm{H}), 2.17$ (ddd, $J=13.9,8.0,4.8 \mathrm{~Hz}, 1 \mathrm{H}), 2.08$ (dt, $J=14.3$, $7.4 \mathrm{~Hz}, 2 \mathrm{H}), 1.02(\mathrm{t}, J=7.2 \mathrm{~Hz}, 3 \mathrm{H})$.

${ }^{13} \mathbf{C}\left\{{ }^{1} \mathbf{H}\right\}$ NMR (151 MHz, DMSO- $\left.d_{6}\right) \delta(\mathrm{ppm}): 199.7,162.5,150.6,128.1,119.8,104.4$, $103.6,96.7,63.6,53.4,49.3,36.7,14.0$.

HRMS (ESI) $m / z$ : Calcd for $\mathrm{C}_{13} \mathrm{H}_{13} \mathrm{Br}_{2} \mathrm{NO}_{3}[\mathrm{M}+\mathrm{H}]^{+}$389.9335; Found 389.9330.

7,10-Dibromo-8-hydroxy-1-propyl-5,6-dihydrobenzo[b]azocine-2,4(1H,3H)-dione (4k)<smiles>CP(C)N1C(=O)CCC(=O)CCc2c(Br)c(O)cc(Br)c21</smiles>

Yellowish oil. The reaction of 2,5-dibromobenzene-1,4-diol (133.95 $\mathrm{mg}, 0.5 \mathrm{mmol})$, 1-propylpiperidin-4-one (105.8 mg, $0.75 \mathrm{mmol})$ and iron (II) phthalocyanine (11.4 mg, $2 \mathrm{mmol} \%$ ), affords $4 \mathbf{k}$ in $67 \%(135.6 \mathrm{mg})$ yield.

${ }^{1} \mathbf{H}$ NMR (400 MHz, $\left.\mathrm{CDCl}_{3}\right) \delta(\mathrm{ppm}): 7.54(\mathrm{~s}, 1 \mathrm{H}), 3.19(\mathrm{q}, J=7.6 \mathrm{~Hz}, 1 \mathrm{H}), 3.13(\mathrm{~d}, J=11.0$ $\mathrm{Hz}, 1 \mathrm{H}), 2.81(\mathrm{~d}, J=11.0 \mathrm{~Hz}, 1 \mathrm{H}), 2.68(\mathrm{q}, J=8.1 \mathrm{~Hz}, 1 \mathrm{H}), 2.53(\mathrm{~d}, J=8.7 \mathrm{~Hz}, 2 \mathrm{H}), 2.36$ (ddd, $J$ $=13.2,7.9,5.0 \mathrm{~Hz}, 1 \mathrm{H}), 2.27(\mathrm{dt}, J=14.2,7.4 \mathrm{~Hz}, 1 \mathrm{H}), 1.57(\mathrm{~d}, J=7.8 \mathrm{~Hz}, 2 \mathrm{H}), 0.94(\mathrm{t}, J=7.4$ $\mathrm{Hz}, 3 \mathrm{H})$.

${ }^{13} \mathbf{C}\left\{{ }^{1} \mathbf{H}\right\}$ NMR (151 MHz, $\left.\mathrm{CDCl}_{3}\right) \delta(\mathrm{ppm}): 200.0,163.8,148.8,128.5,118.9,105.8,103.1$, $96.8,63.4,57.9,53.9,36.4,21.8,11.9$.

HRMS (ESI) $m / z$ : Calcd for $\mathrm{C}_{14} \mathrm{H}_{15} \mathrm{Br}_{2} \mathrm{NO}_{3}[\mathrm{M}+\mathrm{H}]^{+} 403.9491$; Found 403.9488 . 
<smiles>O=C1CCc2c(Br)c(O)cc(Br)c2N(Br)CC1</smiles>

Yellowish oil. The reaction of 2,5-dibromobenzene-1,4-diol (133.95 mg, $0.5 \mathrm{mmol})$, 1-butylpiperidin-4-one (116.5 mg, $0.75 \mathrm{mmol})$ and iron (II) phthalocyanine (11.4 mg, $2 \mathrm{mmol} \%$ ), affords $4 \mathbf{l}$ in $56 \%(117.3 \mathrm{mg})$ yield.

${ }^{1} \mathbf{H}$ NMR $\left(600 \mathrm{MHz}, \mathrm{CDCl}_{3}\right) \delta(\mathrm{ppm}): 7.56(\mathrm{~s}, 1 \mathrm{H}), 3.31-3.24(\mathrm{~m}, 2 \mathrm{H}), 3.23(\mathrm{~d}, J=11.5 \mathrm{~Hz}$, 1H), $2.96(\mathrm{~d}, J=11.5 \mathrm{~Hz}, 1 \mathrm{H}), 2.89-2.83(\mathrm{~m}, 2 \mathrm{H}), 2.74-2.26(\mathrm{~m}, 2 \mathrm{H}), 1.63-1.55(\mathrm{~m}, 2 \mathrm{H}), 1.38$ $(\mathrm{q}, J=7.4 \mathrm{~Hz}, 2 \mathrm{H}), 0.93(\mathrm{t}, J=7.4 \mathrm{~Hz}, 3 \mathrm{H})$.

${ }^{13} \mathbf{C}\left\{{ }^{1} \mathbf{H}\right\}$ NMR (151 MHz, $\left.\mathrm{CDCl}_{3}\right) \delta(\mathrm{ppm}): 202.2,166.4,151.6,128.0,114.1,107.9,95.3$, $62.7,60.9,55.9,53.4,41.0,36.0,14.1$.

HRMS (ESI) $m / z$ : Calcd for $\mathrm{C}_{15} \mathrm{H}_{17} \mathrm{Br}_{2} \mathrm{NO}_{3}[\mathrm{M}+\mathrm{H}]^{+} 417.9648$; Found 417.9645 .

7,10-Dibromo-1-cyclopropyl-8-hydroxy-5,6-dihydrobenzo[b]azocine-2,4(1H,3H)-dione $(4 \mathrm{~m})$<smiles>O=C1CCc2c(Br)c(O)cc(Br)c2N(C2CC2)C1=O</smiles>

Yellowish oil. The reaction of 2,5-dibromobenzene-1,4-diol (133.95 $\mathrm{mg}, 0.5 \mathrm{mmol})$, 1-cyclopropylpiperidin-4-one (104.3 mg, $0.75 \mathrm{mmol})$ and iron (II) phthalocyanine $(11.4 \mathrm{mg}, 2$ mmol\%), affords $4 \mathrm{~m}$ in $55 \%$ (110.8 $\mathrm{mg})$ yield.

${ }^{1} \mathbf{H}$ NMR $\left(400 \mathrm{MHz}, \mathrm{CDCl}_{3}\right) \delta(\mathrm{ppm}): 7.54(\mathrm{~s}, 1 \mathrm{H}), 5.30(\mathrm{~s}, 1 \mathrm{H}), 3.24(\mathrm{dd}, J=15.7,9.6 \mathrm{~Hz}$, 2H), $3.07(\mathrm{~d}, J=11.5 \mathrm{~Hz}, 1 \mathrm{H}), 2.96(\mathrm{q}, J=8.1 \mathrm{~Hz}, 1 \mathrm{H}), 2.41-2.32(\mathrm{~m}, 1 \mathrm{H}), 2.24(\mathrm{dt}, J=13.9$, $7.1 \mathrm{~Hz}, 1 \mathrm{H}), 1.94(\mathrm{q}, J=4.6,3.0 \mathrm{~Hz}, 1 \mathrm{H}), 0.53(\mathrm{dd}, J=23.4,6.1 \mathrm{~Hz}, 4 \mathrm{H})$.

${ }^{13} \mathbf{C}\left\{{ }^{1} \mathbf{H}\right\}$ NMR (151 MHz, $\left.\mathrm{CDCl}_{3}\right) \delta(\mathrm{ppm}):$ 199.6, 163.8, 148.6, 128.3, 118.9, 105.8, 103.0, $97.2,63.4,53.8,36.7,35.6,5.9,5.8$.

HRMS (ESI) $m / z$ : Calcd for $\mathrm{C}_{14} \mathrm{H}_{13} \mathrm{Br}_{2} \mathrm{NO}_{3}[\mathrm{M}+\mathrm{H}]^{+} 401.9335$; Found 401.9331. 


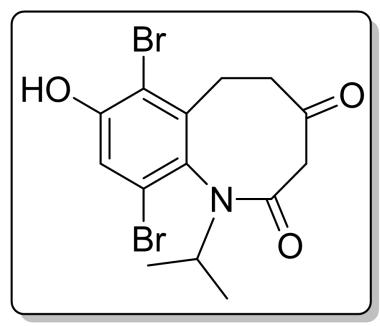

Yellowish oil. The reaction of 2,5-dibromobenzene-1,4-diol (133.95 mg, $0.5 \mathrm{mmol}$ ), 1-isopropylpiperidin-4-one $(105.8 \mathrm{mg}, 0.75 \mathrm{mmol})$ and iron (II) phthalocyanine (11.4 $\mathrm{mg}, 2$ mmol\%), affords $4 \mathrm{n}$ in $60 \%$ (121.5 $\mathrm{mg})$ yield.

${ }^{1}$ H NMR $\left(400 \mathrm{MHz}_{\mathrm{CDCl}}\right) \delta(\mathrm{ppm}): 7.55(\mathrm{~s}, 1 \mathrm{H}), 3.21(\mathrm{dd}, J=15.0,9.1 \mathrm{~Hz}, 2 \mathrm{H}), 2.89(\mathrm{~d}, J$ $=11.1 \mathrm{~Hz}, 1 \mathrm{H}), 2.74(\mathrm{~d}, J=8.2 \mathrm{~Hz}, 1 \mathrm{H}), 2.64-2.53(\mathrm{~m}, 1 \mathrm{H}), 2.34(\mathrm{dd}, J=7.8,5.2 \mathrm{~Hz}, 1 \mathrm{H}), 2.31$ $-2.20(\mathrm{~m}, 1 \mathrm{H}), 1.16(\mathrm{dd}, J=9.7,6.4 \mathrm{~Hz}, 6 \mathrm{H})$.

${ }^{13} \mathbf{C}\left\{{ }^{1} \mathbf{H}\right\}$ NMR (151 MHz, $\left.\mathrm{CDCl}_{3}\right) \delta(\mathrm{ppm}): 199.8,163.8,148.8,128.6,118.9,105.8,103.3$, $96.6,61.7,54.7,51.6,36.4,21.3$.

HRMS (ESI) $m / z$ : Calcd for $\mathrm{C}_{14} \mathrm{H}_{15} \mathrm{Br}_{2} \mathrm{NO}_{3}[\mathrm{M}+\mathrm{H}]^{+} 403.9419$; Found 403.9418 .

7,10-Dibromo-8-hydroxy-1,3-dimethyl-5,6-dihydrobenzo $[b]$ azocine-2,4(1H,3H)-dione (4p)<smiles>CC1C(=O)CCc2c(Br)c(O)cc(Br)c2N1C</smiles>

Yellowish oil. The reaction of 2,5-dibromobenzene-1,4-diol (133.95 mg, $0.5 \mathrm{mmol}$ ), 1,3-dimethylpiperidin-4-one (95 mg, $0.75 \mathrm{mmol}$ ) and iron (II) phthalocyanine (11.4 mg, 2 mmol\%), affords $4 p$ in $31 \%(60.2 \mathrm{mg})$ yield.

${ }^{1}$ H NMR $\left(400 \mathrm{MHz}, \mathrm{CDCl}_{3}\right) \delta(\mathrm{ppm}): 7.54(\mathrm{~s}, 1 \mathrm{H}), 5.31(\mathrm{~d}, J=0.5 \mathrm{~Hz}, 1 \mathrm{H}), 3.22(\mathrm{~d}, J=11.3$ $\mathrm{Hz}, 1 \mathrm{H}), 3.10-3.04(\mathrm{~m}, 1 \mathrm{H}), 2.95(\mathrm{~d}, J=11.4 \mathrm{~Hz}, 1 \mathrm{H}), 2.77-2.65(\mathrm{~m}, 2 \mathrm{H}), 2.49(\mathrm{~s}, 3 \mathrm{H}), 0.92(\mathrm{~d}$, $J=6.7 \mathrm{~Hz}, 3 \mathrm{H})$.

${ }^{13} \mathbf{C}\left\{{ }^{1} \mathbf{H}\right\}$ NMR (151 MHz, $\left.\mathrm{CDCl}_{3}\right) \delta(\mathrm{ppm}): 199.3,164.0,148.9,128.6,119.7,105.6,103.0$, 98.6, 64.7, 62.7, 43.7, 42.6, 11.1.

HRMS (ESI) $m / z$ : Calcd for $\mathrm{C}_{13} \mathrm{H}_{13} \mathrm{Br}_{2} \mathrm{NO}_{3}[\mathrm{M}+\mathrm{H}]^{+}$388.9262; Found 388.9256 . 


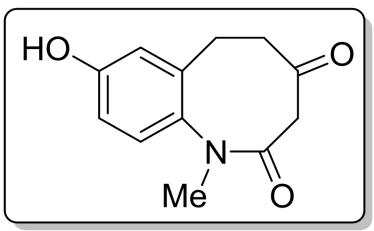

Yellowish oil. The reaction of hydroquinone ( $55 \mathrm{mg}, 0.5 \mathrm{mmol}), 1$-methylpiperidin-4-ol (86 $\mathrm{mg}, 0.75 \mathrm{mmol}$ ) and iron (II) phthalocyanine (11.4 mg, $2 \mathrm{mmol} \%$ ), affords $4 q$ in $34 \%$ (37.2 $\mathrm{mg}$ ) yield.

${ }^{1} \mathbf{H}$ NMR $\left(600 \mathrm{MHz}, \mathrm{CDCl}_{3}\right) \delta(\mathrm{ppm}): 7.23(\mathrm{dd}, J=8.9,2.8 \mathrm{~Hz}, 1 \mathrm{H}), 7.08(\mathrm{~d}, J=2.7 \mathrm{~Hz}, 1 \mathrm{H})$, $6.99(\mathrm{dd}, J=8.8,2.3 \mathrm{~Hz}, 1 \mathrm{H}), 3.22(\mathrm{td}, J=9.3,8.7,4.9 \mathrm{~Hz}, 1 \mathrm{H}), 3.08(\mathrm{~d}, J=10.8 \mathrm{~Hz}, 1 \mathrm{H}), 2.85-$ $2.79(\mathrm{~m}, 1 \mathrm{H}), 2.70(\mathrm{q}, J=8.3 \mathrm{~Hz}, 1 \mathrm{H}), 2.50(\mathrm{~s}, 3 \mathrm{H}), 2.38$ (ddd, $J=13.6,8.4,4.9 \mathrm{~Hz}, 1 \mathrm{H}), 2.27-$ $2.21(\mathrm{~m}, 1 \mathrm{H})$

${ }^{13} \mathbf{C}\left\{{ }^{1} \mathbf{H}\right\}$ NMR $\left(151 \mathrm{MHz}, \mathrm{CDCl}_{3}\right) \delta$ (ppm): 202.1, 166.3, 152.0, 128.2, 120.2, 114.1, 108.0, $95.5,65.0,55.7,41.9,36.5$.

HRMS (ESI) $m / z$ : Calcd for $\mathrm{C}_{12} \mathrm{H}_{13} \mathrm{NO}_{3}[\mathrm{M}+\mathrm{H}]^{+}$220.0968; Found 220.0966.

\subsection{Turn on / off experiments}

The relationship between reaction conditions and light time was determined by turn on / off experiment. Using the above mentioned general procedure, hydroquinone 1a $(0.5 \mathrm{mmol})$, N-methyl-4-pipertidinone 2a (0.75 mmol), iron (II) phthalocyanine (11.4 mg, 2 mmol\%), HOAc $(1.0 \mathrm{~mL})$ and water $(2.0 \mathrm{ml})$ were added to the reaction vessel one by one. The reaction was carried out under alternately irradiation by a commercially available $5 \mathrm{~W}$ white LEDs or dark.

\subsection{Radical capture experiment by butylated hydroxytoluene (BHT)}

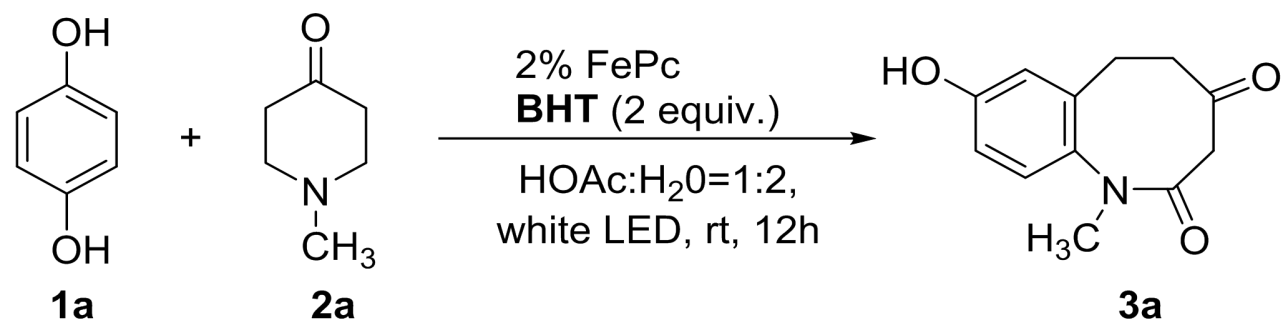

Iron (II) phthalocyanine (11.4 mg, 2 mmol\%), butylated hydroxytoluene (BHT) (2 equiv), hydroquinone 1a $(0.5 \mathrm{mmol})$, N-methyl-4-pipertidinone 2a $(0.75 \mathrm{mmol})$, HOAc $(1.0 \mathrm{~mL})$ and 
water $(2.0 \mathrm{~mL})$, air, $5 \mathrm{~W}$ white LEDs, $24 \mathrm{~h}$. In this case, no reaction occurred. Adducts of N-methylpiperidinone with BHT were detected by ESI-HRMS.

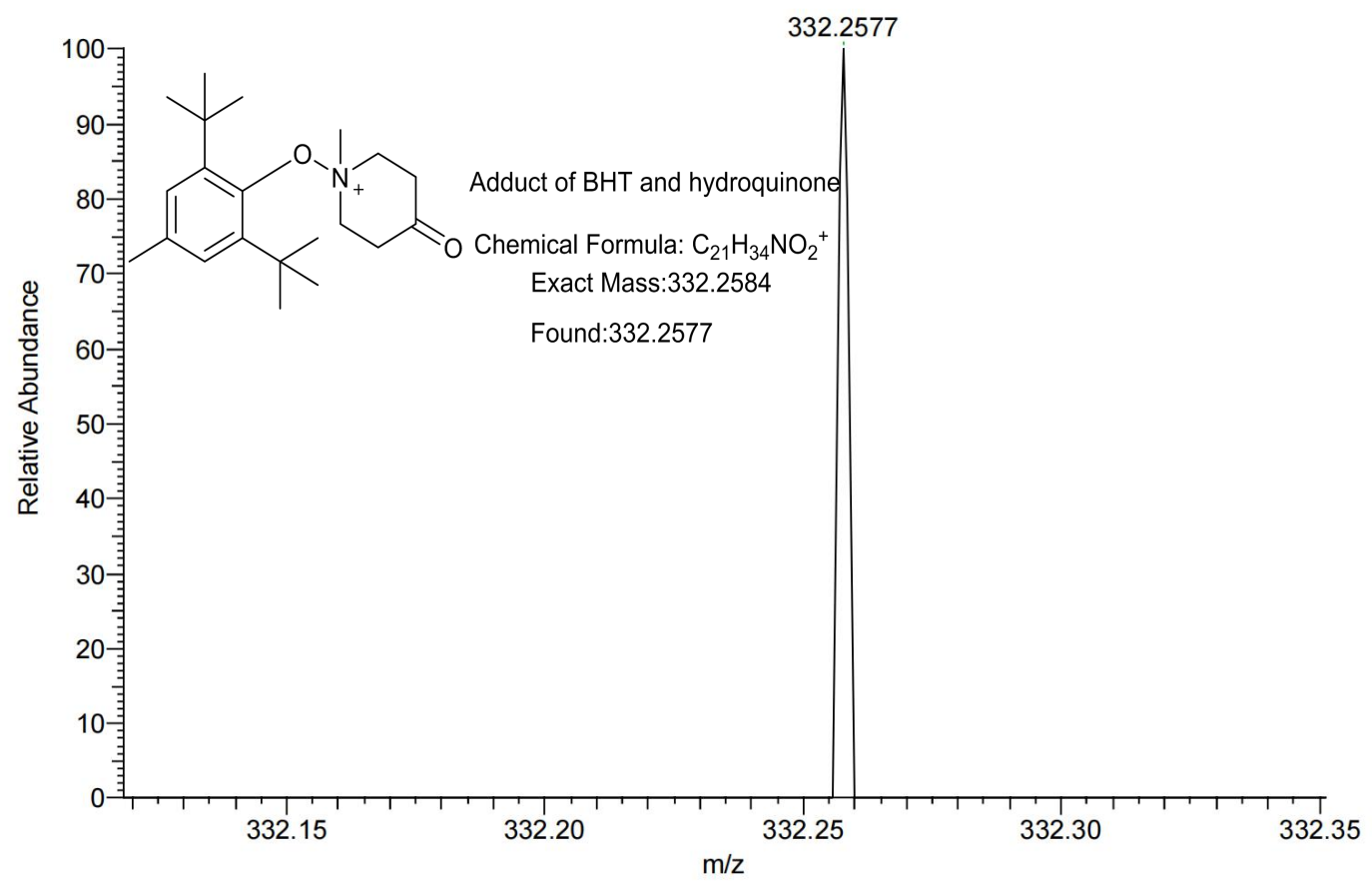

Figure S2. HRMS spectrum of the adduct of BHT and N-methylpiperidinone

\subsection{EPR tests}

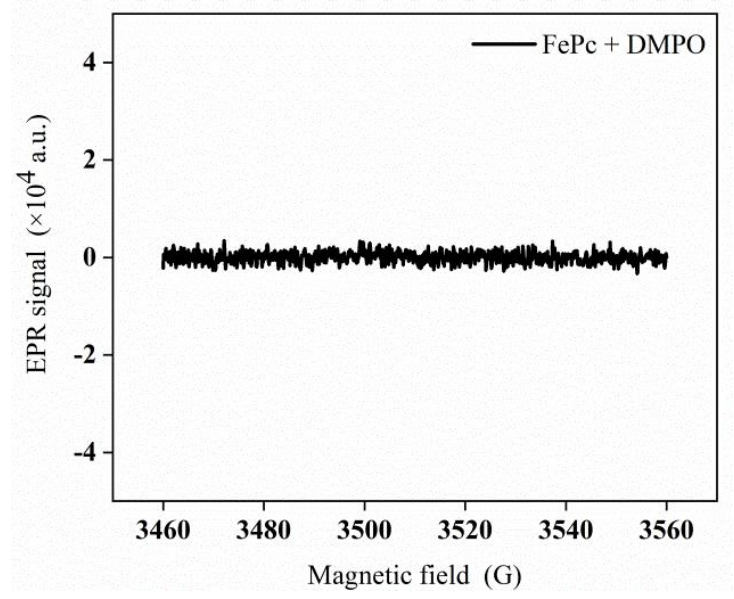

Figure S3. Electron spin resonance (EPR) spectrum: a solution of DMPO $\left(4 \times 10^{-2} \mathrm{M}\right)$ and $\mathrm{FeFc}\left(4 \times 10^{-4} \mathrm{M}\right)$ in air-saturated $\mathrm{HOAc} / \mathrm{H}_{2} \mathrm{O}(1 / 2, \mathrm{v} / \mathrm{v})$ with irradiation. 


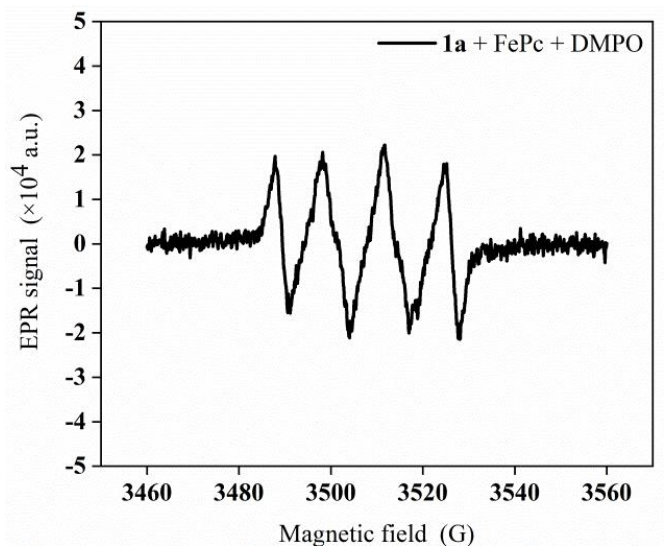

Figure S4. Electron spin resonance (EPR) spectrum: a solution of hydroquinone $\left(4 \times 10^{-2} \mathrm{M}\right)$, DMPO $\left(4 \times 10^{-2}\right)$ and $\mathrm{FeFc}\left(4 \times 10^{-4} \mathrm{M}\right)$ in air-saturated $\mathrm{HOAc} / \mathrm{H}_{2} \mathrm{O}(1 / 2, \mathrm{v} / \mathrm{v})$ with $5 \mathrm{~W}$ white LED irradiation.

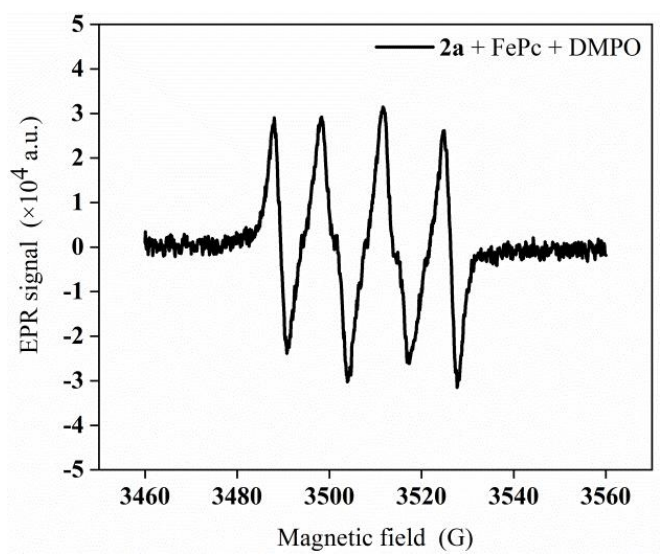

Figure S5. Electron spin resonance (EPR) spectrum: a solution of N-methyl-4-piperidinone $\left(4 \times 10^{-2} \mathrm{M}\right)$, DMPO $\left(4 \times 10^{-2}\right.$ and $\mathrm{FeFc}\left(4 \times 10^{-4} \mathrm{M}\right)$ in air-saturated $\mathrm{HOAc} / \mathrm{H}_{2} \mathrm{O}(1 / 2, \mathrm{v} / \mathrm{v})$ with $5 \mathrm{~W}$ white LED irradiation.

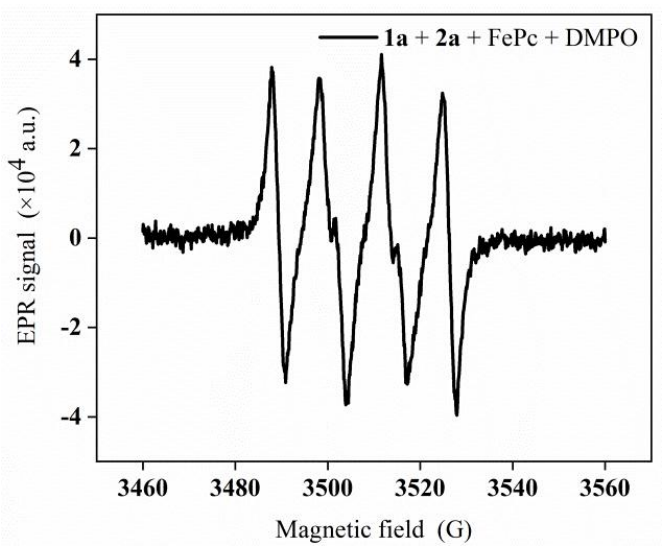

Figure S6. Electron spin resonance (EPR) spectrum: a solution of hydroquinone $\left(4 \times 10^{-2} \mathrm{M}\right)$, N-methyl-4-piperidinone $\left(4 \times 10^{-2} \mathrm{M}\right)$, DMPO $\left(4 \times 10^{-2}\right.$ and $\mathrm{FeFc}\left(4 \times 10^{-4} \mathrm{M}\right)$ in air-saturated $\mathrm{HOAc} / \mathrm{H}_{2} \mathrm{O}(1 / 2, \mathrm{v} / \mathrm{v})$ with $5 \mathrm{~W}$ white LED irradiation. 


\subsection{Fluorescence quenching experiments}

Quenched by hydroquinone 1a: For each quenching experiment, the emission intensity of the solution $(1 \mathrm{~mL})$ of photocatalyst FePc with different concentration of quencher $(0,0.5,1.0,1.5,2.0 \mathrm{mM})$ was collected. As shown in Figure S7, hydroquinone 1a was capable of quenching the excited state of photocatalyst FePc.

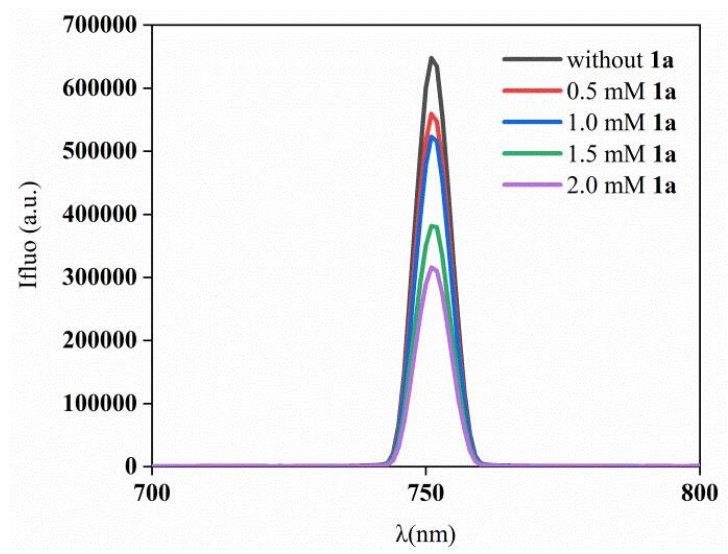

Figure S7. Fluorescence spectra of a solution of photocatalyst $\mathrm{FePc}$ in $\mathrm{AcOH}$ containing 0 (black), $0.5 \mathrm{mM}$ (red), $1.0 \mathrm{mM}$ (blue), $1.5 \mathrm{mM}$ (green), $2.0 \mathrm{mM}$ purple) of hydroquinone 1a. $\lambda_{\mathrm{ex}}=$ $376 \mathrm{~nm}, \lambda_{\mathrm{em}}=751 \mathrm{~nm}$.

Quenched by $N$-methyl-4-piperidinone 2a: For each quenching experiment, the emission intensity of the solution $(1 \mathrm{~mL})$ of photocatalyst FePc with different concentration of quencher $(0,0.5,1.0,1.5,2.0 \mathrm{mM})$ was collected. As shown in Figure S8, $N$-methyl-4-piperidinone 2a was capable of quenching the excited state of photocatalyst FePc.



Figure S8. Fluorescence spectra of a solution of photocatalyst $\mathrm{FePc}$ in $\mathrm{AcOH}$ containing 0 (black), $\quad 0.5 \mathrm{mM}$ (red), $\quad 1.0 \mathrm{mM}$ (blue), $1.5 \mathrm{mM}$ (green), $2.0 \mathrm{mM}$ purple) of $\mathrm{N}$-methyl-4-piperidinone 2a. $\lambda_{\mathrm{ex}}=376 \mathrm{~nm}, \lambda_{\mathrm{em}}=751 \mathrm{~nm}$. 


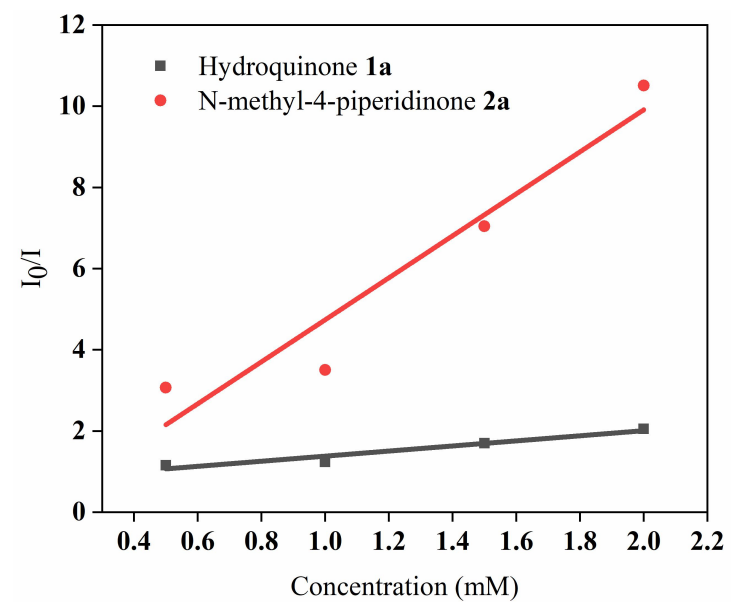

Figure S9. Stern-Volmer plots. $\mathrm{I}_{0}$ and I are respective luminescence intensities in the absence and presence of the indicated concentrations of the corresponding quencher.

\subsection{Cyclic voltammetry (CV) experiments}

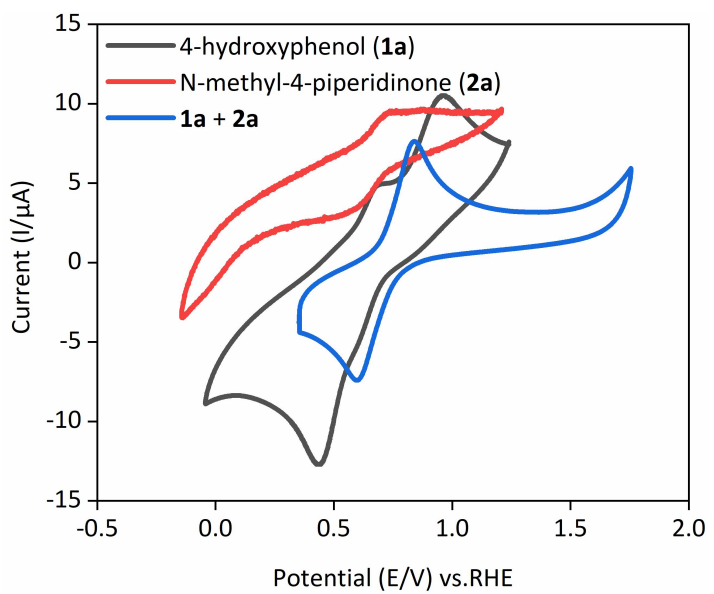

Figure S10. Cyclic voltammogram at $100 \mathrm{mV} \mathrm{s}^{-1}$ scan rate a reversible hydrogen electrode in $\mathrm{AcONa} / \mathrm{AcOH}$ buffer solution $(1 \mathrm{mM}, \mathrm{pH}=4.4) ; \mathbf{1 a}(1 \mathrm{mM}$, black $) ; \mathbf{2 a}(1 \mathrm{mM}$, red $)$ and $\mathbf{1 a}+\mathbf{2 a}(1$ $\mathrm{mM}$, blue). 


\section{Copies of NMR spectra of compounds}

8-Hydroxy-1-methyl-5,6-dihydrobenzo $[b]$ azocine-2,4(1H,3H)-dione (3a)<smiles>CN1C(=O)CCCc2cc(O)ccc21</smiles>

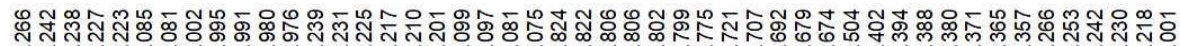

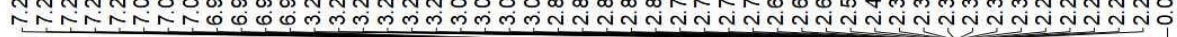
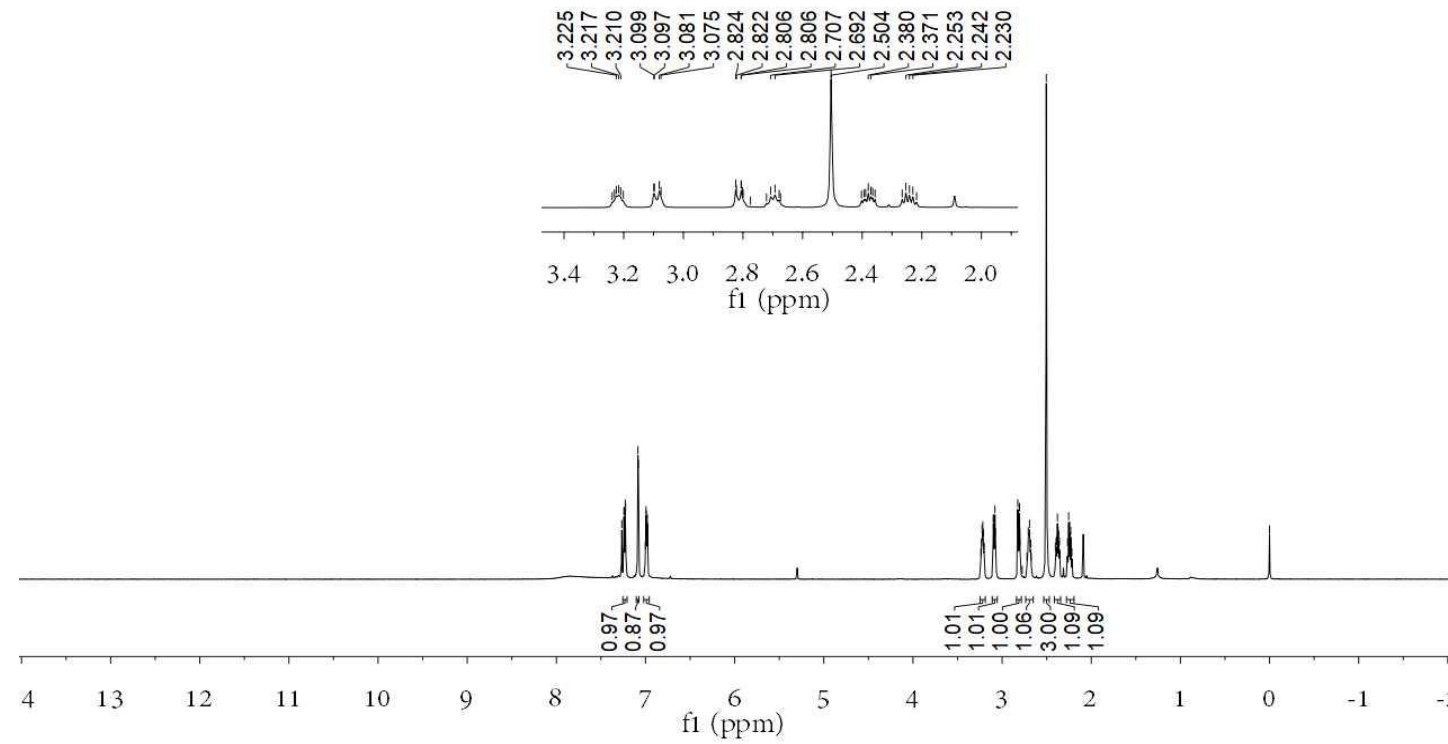

\begin{tabular}{|c|c|c|c|c|c|c|c|}
\hline $\begin{array}{l}\text { O̊ } \\
\text { đू. }\end{array}$ & 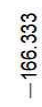 & $\frac{1}{\stackrel{1}{5}}$ &  & 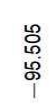 & 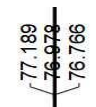 & 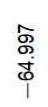 & $\begin{array}{l}\text { R } \\
6 \\
6 \\
0 \\
0 \\
1\end{array}$ \\
\hline
\end{tabular}

$\begin{array}{lllllllllllllllllllllllll}230 & 220 & 210 & 200 & 190 & 180 & 170 & 160 & 150 & 140 & 130 & 120 & 110 & 100 & 90 & 80 & 70 & 60 & 50 & 40 & 30 & 20 & 10 & 0 & -10\end{array}$ f1 (ppm)

Figure S11. ${ }^{1} \mathrm{H}(600 \mathrm{M})$ and ${ }^{13} \mathrm{C}\left\{{ }^{1} \mathrm{H}\right\}(151 \mathrm{M})$ NMR spectra of 3a in $\mathrm{CDCl}_{3}$. 


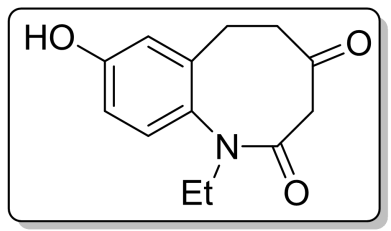

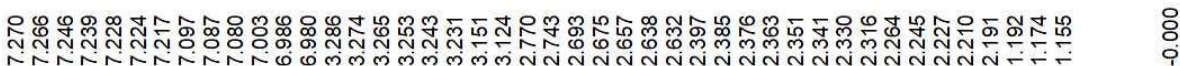

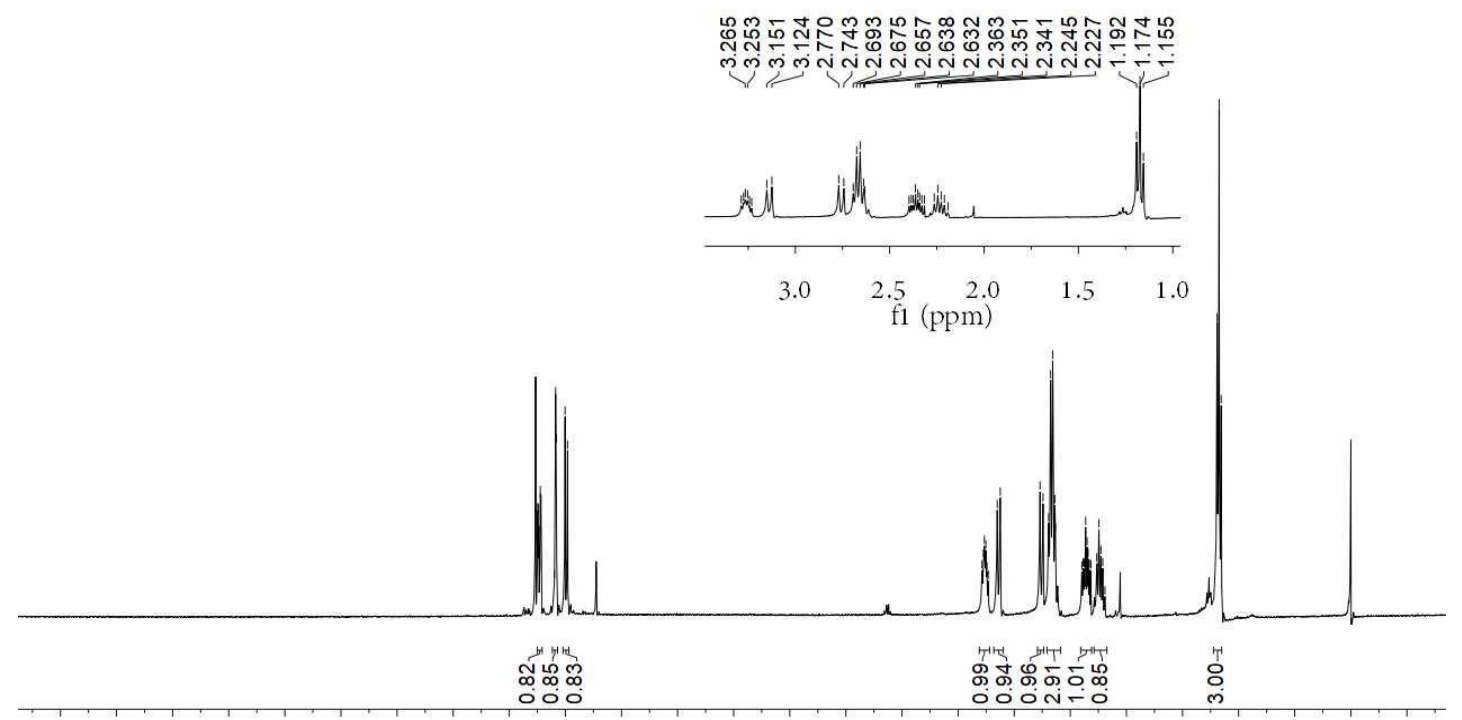

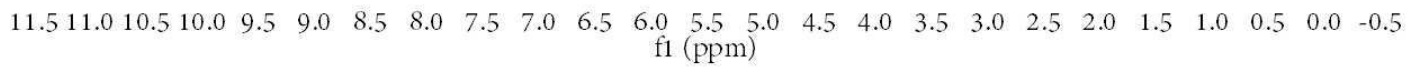

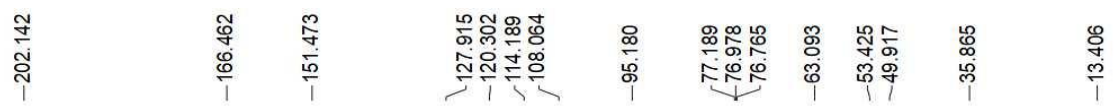



Figure S12. ${ }^{1} \mathrm{H}(400 \mathrm{M})$ and ${ }^{13} \mathrm{C}\left\{{ }^{1} \mathrm{H}\right\}(151 \mathrm{M}) \mathrm{NMR}$ spectra of $3 \mathrm{~b}$ in $\mathrm{CDCl}_{3}$ 


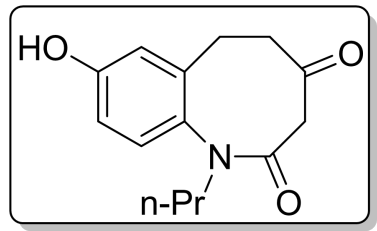

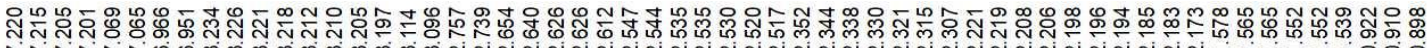

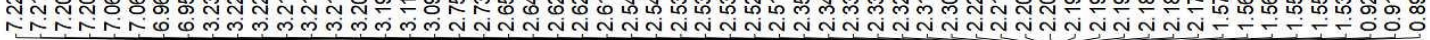
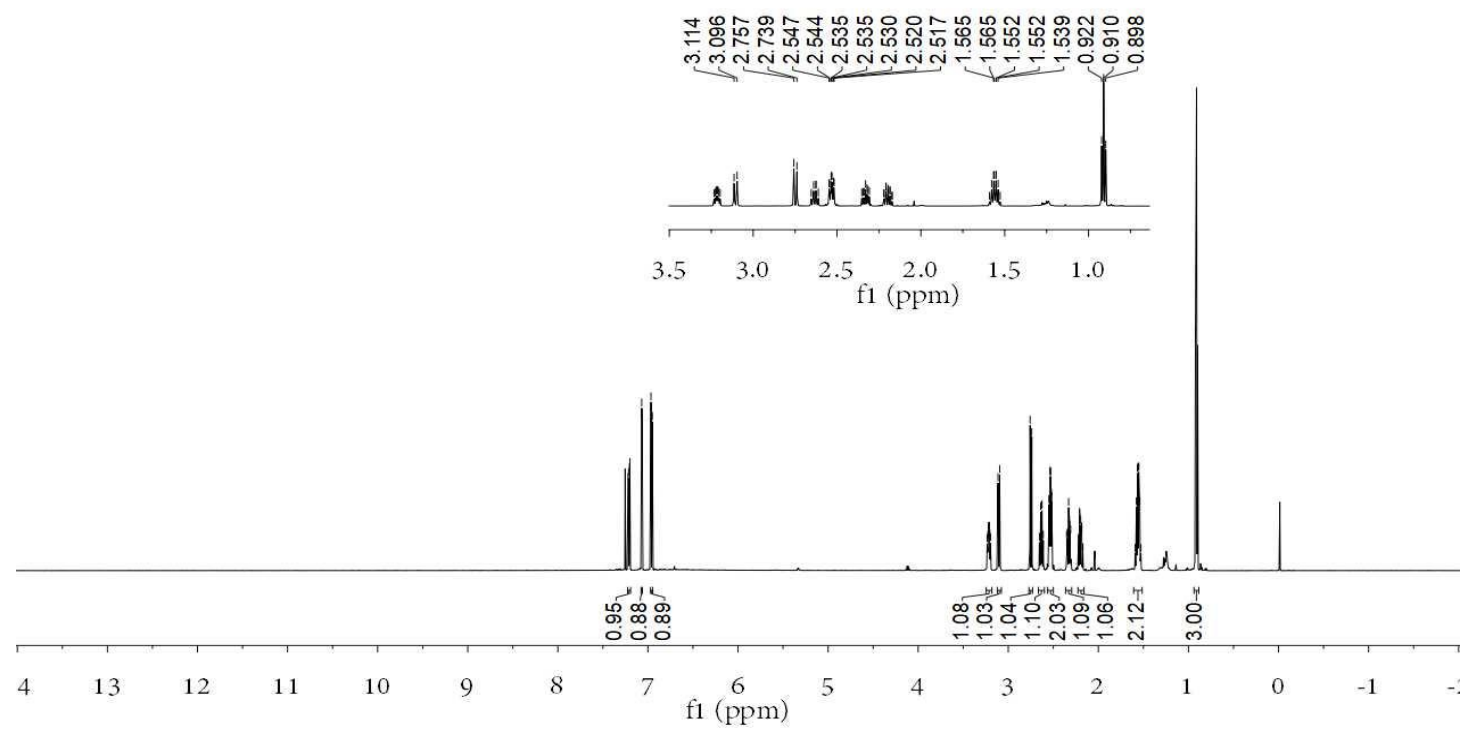

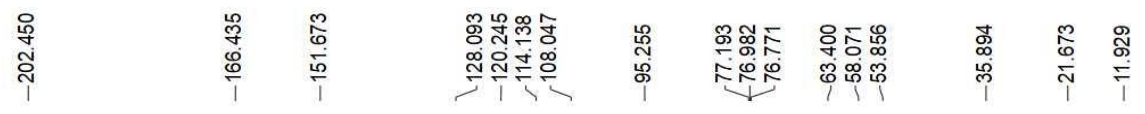



Figure S13. ${ }^{1} \mathrm{H}(600 \mathrm{M})$ and ${ }^{13} \mathrm{C}\left\{{ }^{1} \mathrm{H}\right\}(151 \mathrm{M})$ NMR spectra of $3 \mathrm{c}$ in $\mathrm{CDCl}_{3}$ 


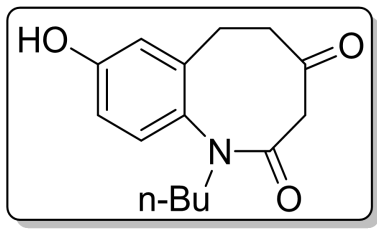

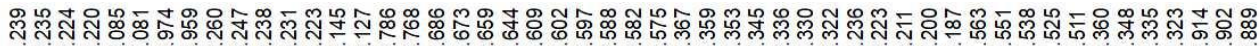
NTN

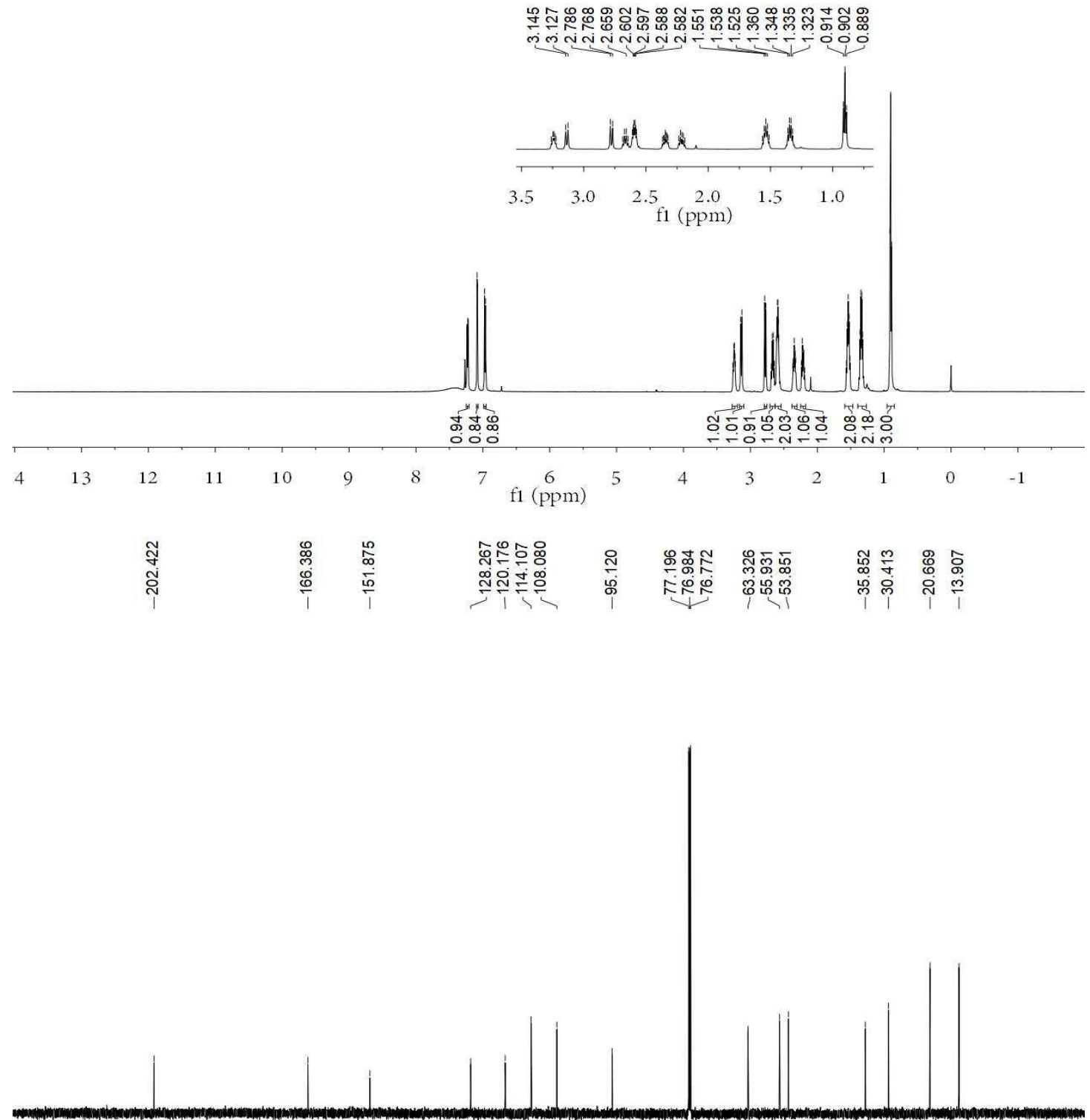

$\begin{array}{lllllllllllllllllllllllll}230 & 220 & 210 & 200 & 190 & 180 & 170 & 160 & 150 & 140 & 130 & 120 & 110 & 100 & 90 & 80 & 70 & 60 & 50 & 40 & 30 & 20 & 10 & 0 & -10\end{array}$ f1 (ppm)

Figure S14. ${ }^{1} \mathrm{H}(600 \mathrm{M})$ and ${ }^{13} \mathrm{C}\left\{{ }^{1} \mathrm{H}\right\}(151 \mathrm{M})$ NMR spectra of $3 \mathrm{~d}$ in $\mathrm{CDCl}_{3}$ 


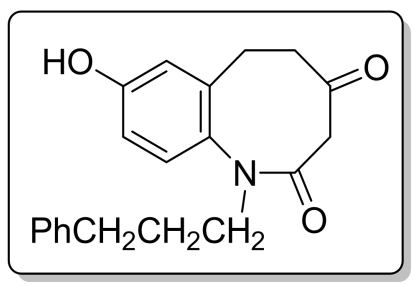

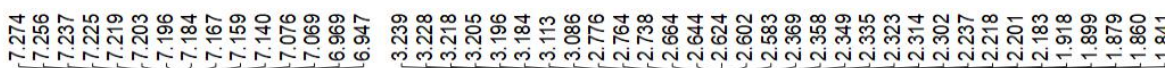
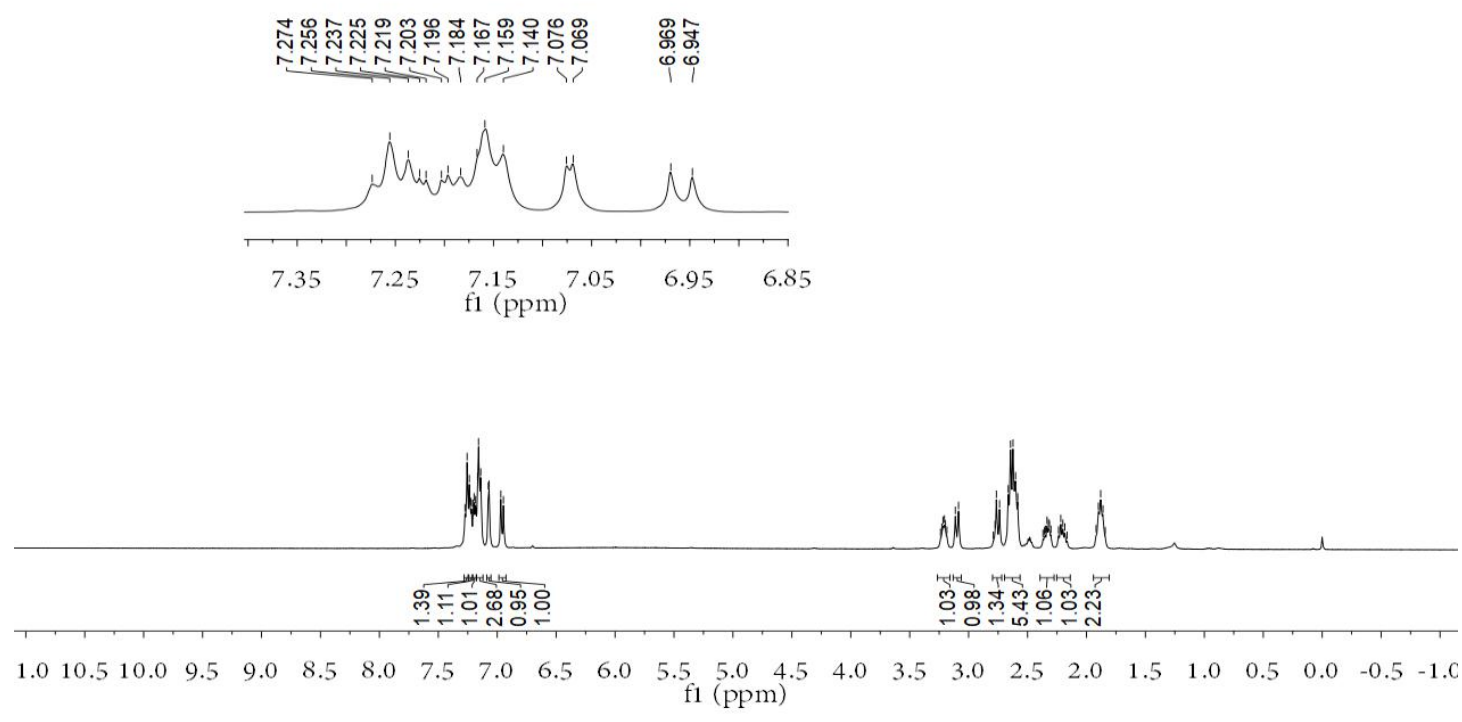

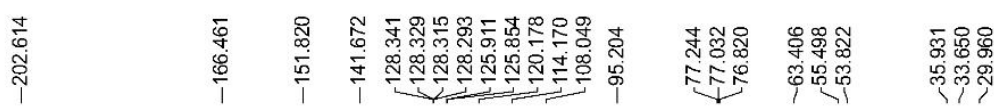

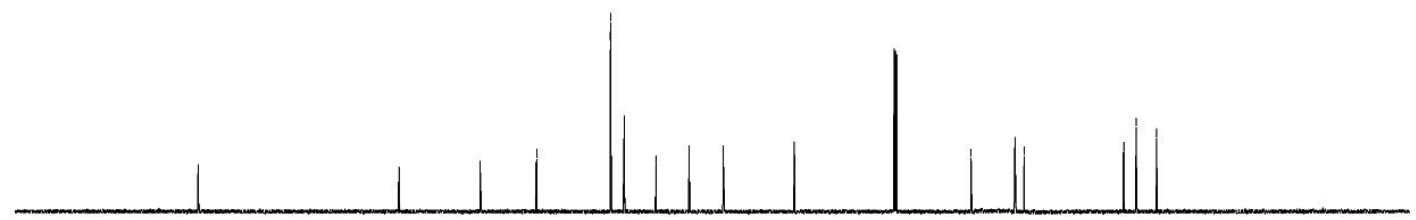

$\begin{array}{llllllllllllllllllllllllll}230 & 220 & 210 & 200 & 190 & 180 & 170 & 160 & 150 & 140 & 130 & 120 & 110 & 100 & 90 & 80 & 70 & 60 & 50 & 40 & 30 & 20 & 10 & 0 & -10\end{array}$ f1 (ppm)

Figure S15. ${ }^{1} \mathrm{H}(400 \mathrm{M})$ and ${ }^{13} \mathrm{C}\left\{{ }^{1} \mathrm{H}\right\}(151 \mathrm{M})$ NMR spectra of $3 \mathrm{e}$ in $\mathrm{CDCl}_{3}$ 


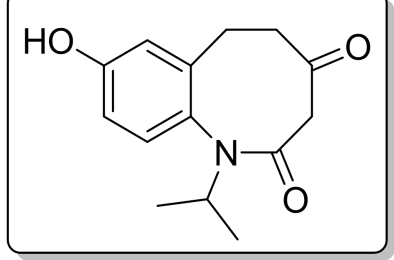

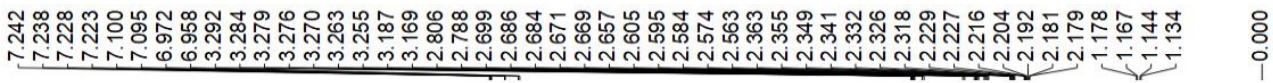

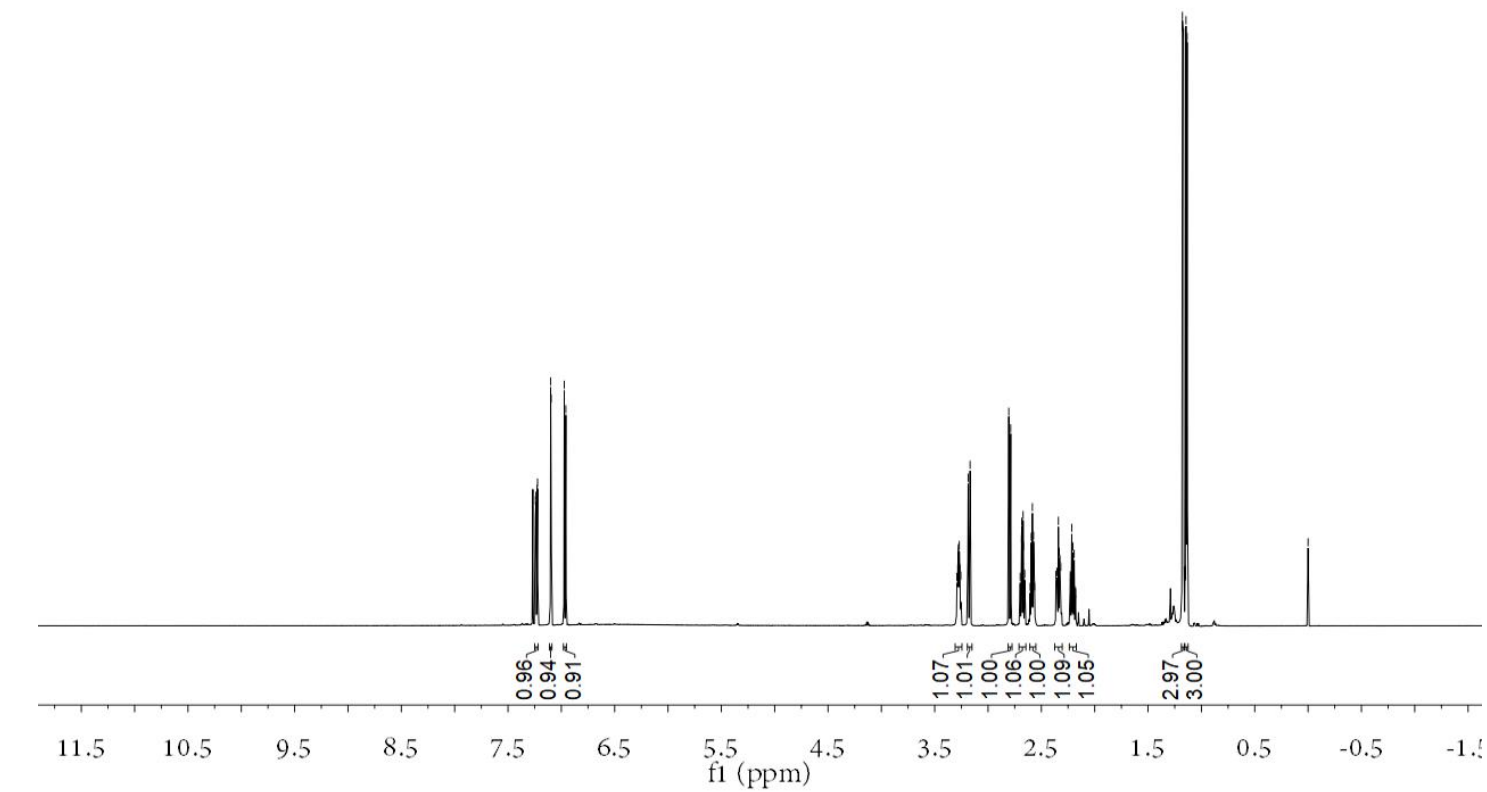

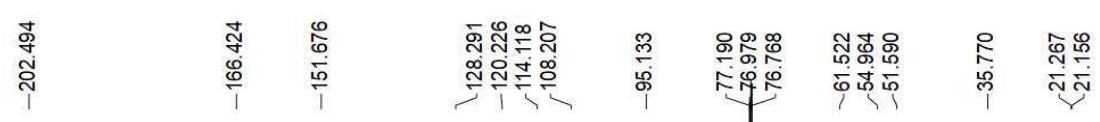

$\begin{array}{llllllllllllllllllllllllllll}230 & 220 & 210 & 200 & 190 & 180 & 170 & 160 & 150 & 140 & 130 & 120 & 110 & 100 & 90 & 80 & 70 & 60 & 50 & 40 & 30 & 20 & 10 & 0 & -10\end{array}$ f1 (ppm)

Figure S16. ${ }^{1} \mathrm{H}(600 \mathrm{M})$ and ${ }^{13} \mathrm{C}\left\{{ }^{1} \mathrm{H}\right\}(151 \mathrm{M})$ NMR spectra of $3 \mathrm{f}$ in $\mathrm{CDCl}_{3}$ 


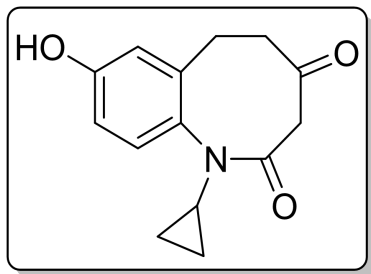

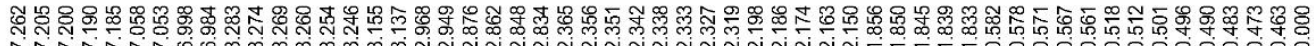

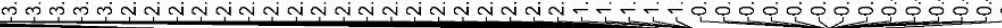



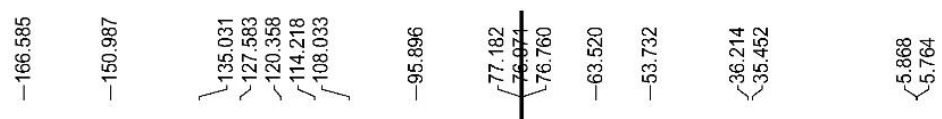

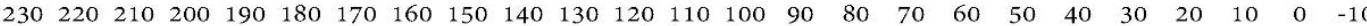
fl $(\mathrm{ppm})$

Figure S17. ${ }^{1} \mathrm{H}(600 \mathrm{M})$ and ${ }^{13} \mathrm{C}\left\{{ }^{1} \mathrm{H}\right\}(151 \mathrm{M})$ NMR spectra of $3 \mathrm{~h}$ in $\mathrm{CDCl}_{3}$ 
<smiles>O=C1CCc2cc(O)ccc2N(Cc2ccccc2)C1=O</smiles>

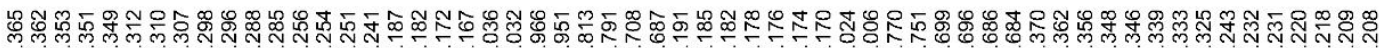

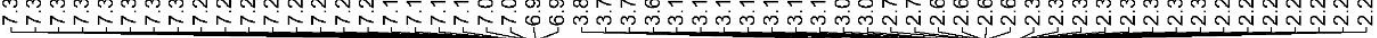

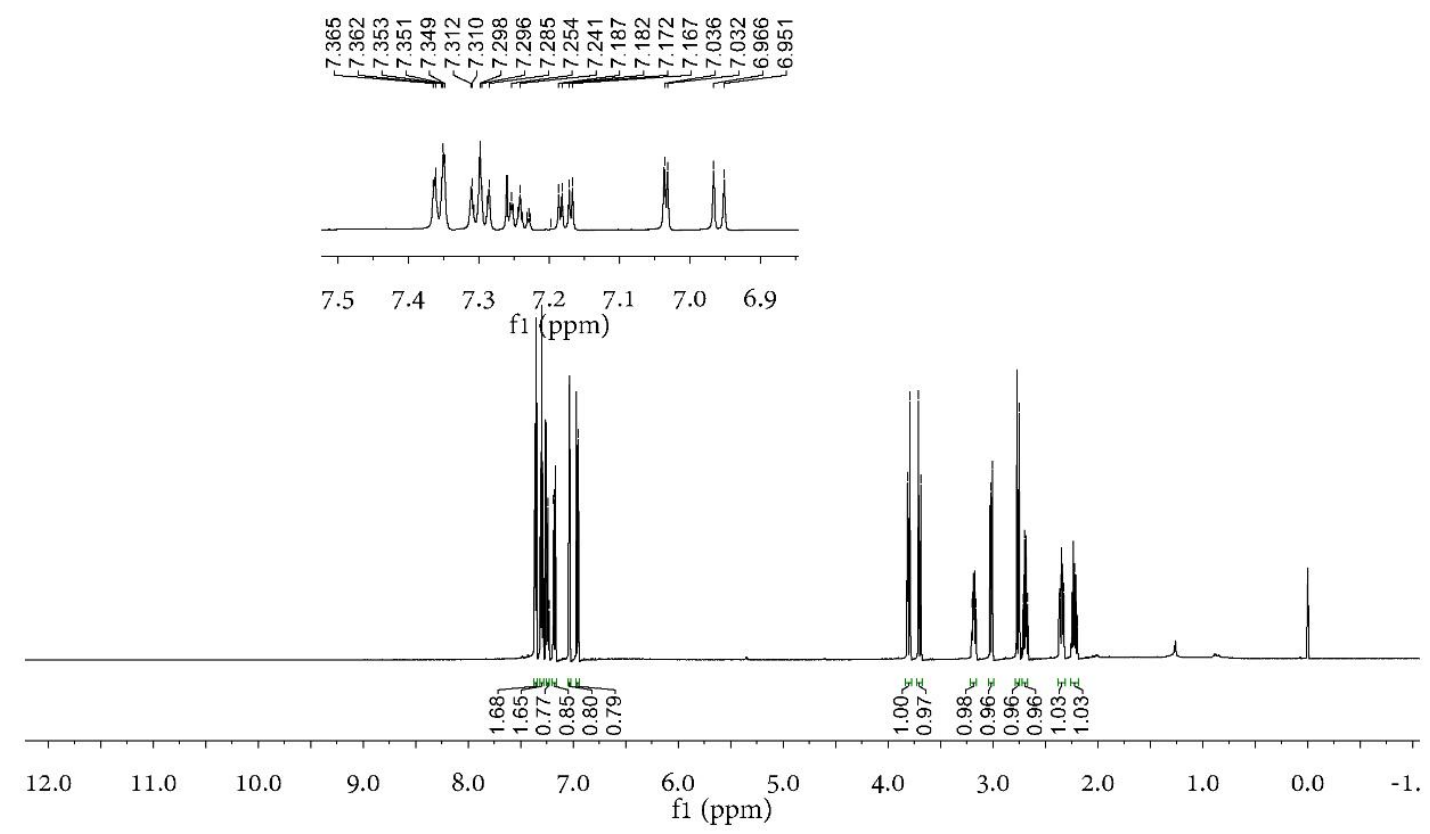

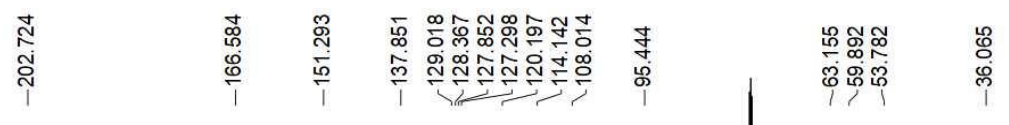

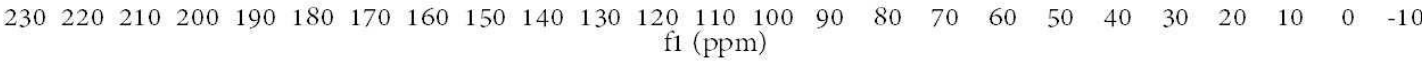

Figure S18. ${ }^{1} \mathrm{H}(600 \mathrm{M})$ and ${ }^{13} \mathrm{C}\left\{{ }^{1} \mathrm{H}\right\}(151 \mathrm{M})$ NMR spectra of $3 \mathrm{i}$ in $\mathrm{CDCl}_{3}$ 
<smiles>C=CC1C=CC(O)=CC=C2C(CCC(=O)CC(=O)N2C)C1</smiles>


-

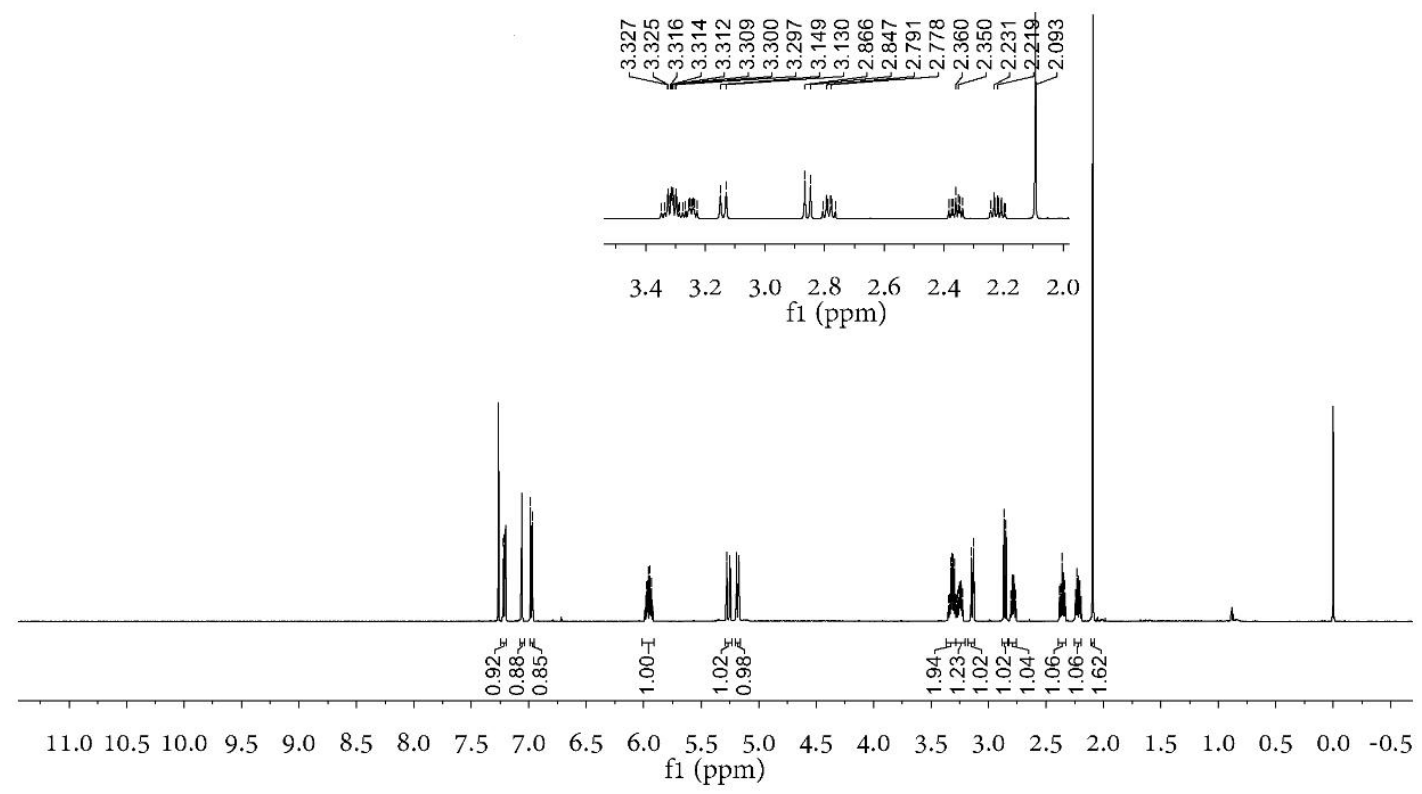

Figure S19. ${ }^{1} \mathrm{H}(600 \mathrm{M})$ and ${ }^{13} \mathrm{C}\left\{{ }^{1} \mathrm{H}\right\}(151 \mathrm{M})$ NMR spectra of $3 \mathrm{j}$ in $\mathrm{CDCl}_{3}$ 
<smiles>C#CCN1C(=O)C(=O)CCc2cc(O)ccc21</smiles>

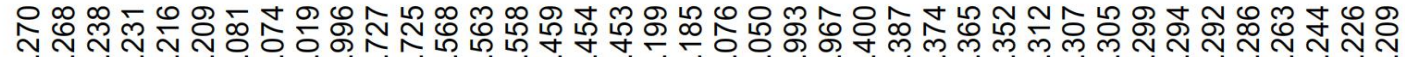



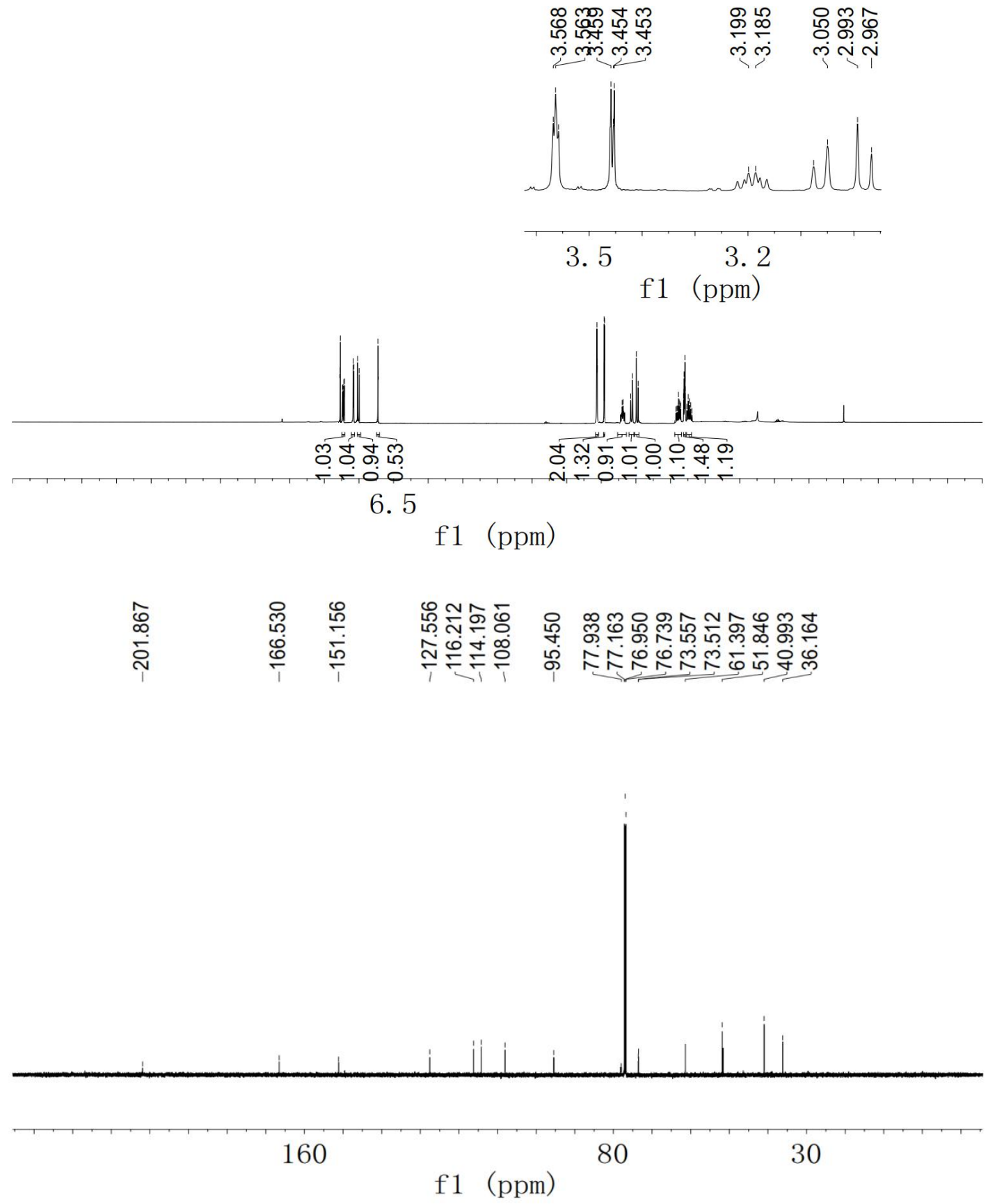

Figure S20. ${ }^{1} \mathrm{H}(400 \mathrm{M})$ and ${ }^{13} \mathrm{C}\left\{{ }^{1} \mathrm{H}\right\}(151 \mathrm{M})$ NMR spectra of $3 \mathrm{~K}$ in $\mathrm{CDCl}_{3}$ 
Ethyl-2-(8-hydroxy-2,4-dioxo-3,4,5,6-tetrahydrobenzo[b]azocin-1(2H)-yl)acetate (3I)<smiles>CCOC(=O)CN1C(=O)CCC2CCCCC21</smiles>

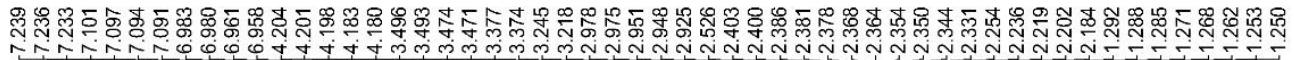

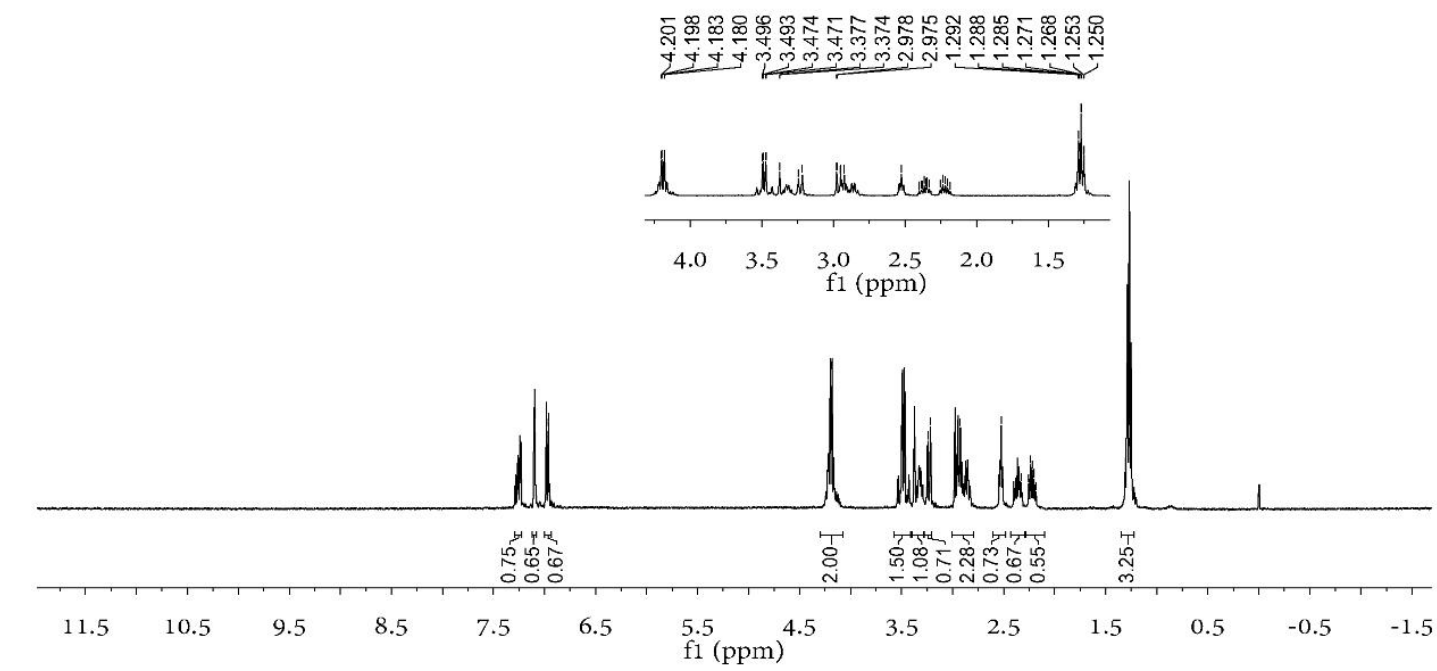



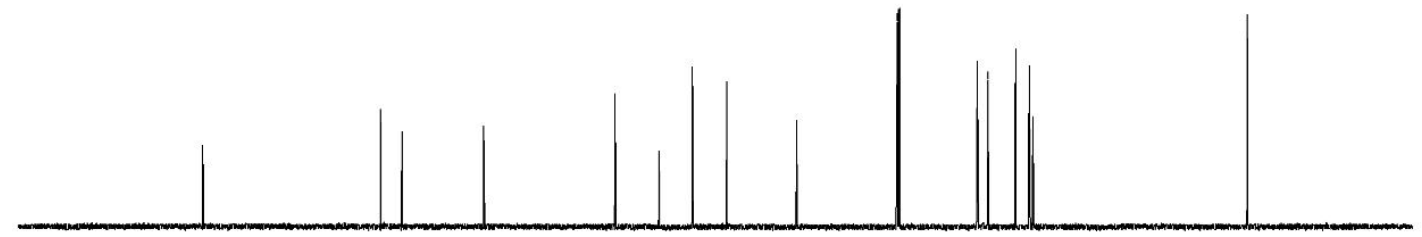

$\begin{array}{lllllllllllllllllllllllll}230 & 220 & 210 & 200 & 190 & 180 & 170 & 160 & 150 & 140 & 130 & 120 & 110 & 100 & 90 & 80 & 70 & 60 & 50 & 40 & 30 & 20 & 10 & 0 & -10\end{array}$ f1 (ppm)

Figure S21. ${ }^{1} \mathrm{H}(400 \mathrm{M})$ and ${ }^{13} \mathrm{C}\left\{{ }^{1} \mathrm{H}\right\}(151 \mathrm{M}) \mathrm{NMR}$ spectra of 31 in $\mathrm{CDCl}_{3}$ 
8-Hydroxy-1,9-dimethyl-5,6-dihydrobenzo[b]azocine-2,4(1H,3H)-dione (4a) and

8-Hydroxy-1,7-dimethyl-5,6-dihydrobenzo[b]azocine-2,4(1H,3H)-dione(4a')

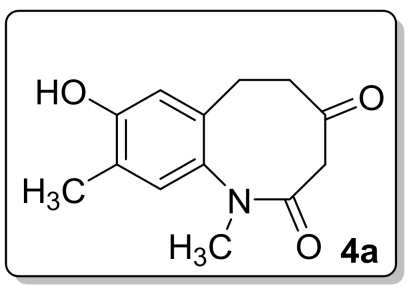

and

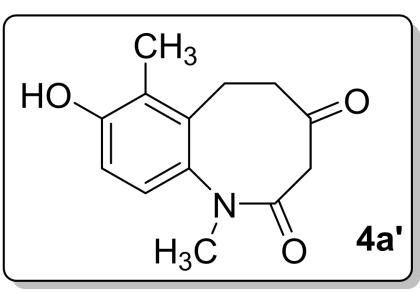

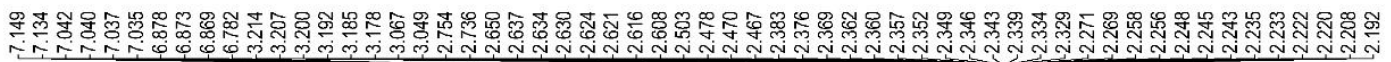

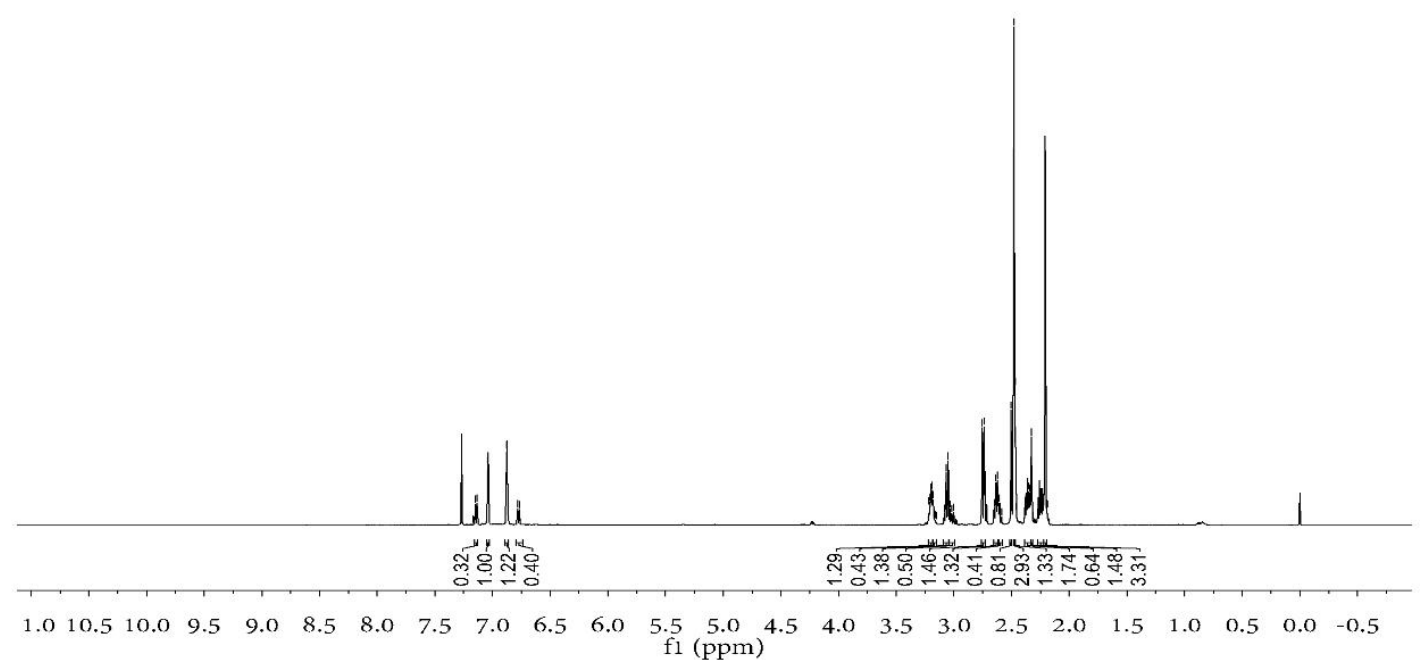

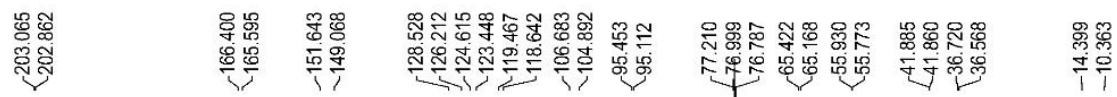

$\begin{array}{lllllllllllllllllllllllll}230 & 220 & 210 & 200 & 190 & 180 & 170 & 160 & 150 & 140 & 130 & 120 & 110 & 100 & 90 & 80 & 70 & 60 & 50 & 40 & 30 & 20 & 10 & 0 & -10\end{array}$

f1 $(\mathrm{ppm})$

Figure S22. ${ }^{1} \mathrm{H}(600 \mathrm{M})$ and ${ }^{13} \mathrm{C}\left\{{ }^{1} \mathrm{H}\right\}(151 \mathrm{M}) \mathrm{NMR}$ spectra of $4 \mathrm{a}$ and $4 \mathrm{a}^{\prime}$ in $\mathrm{CDCl}_{3}$ 
7-Chloro-8-hydroxy-1-methyl-5,6-dihydrobenzo[b]azocine-2,4(1H,3H)-dione (4b)<smiles>CN1C(=O)CCCCCC1=O</smiles>

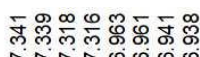



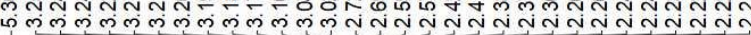

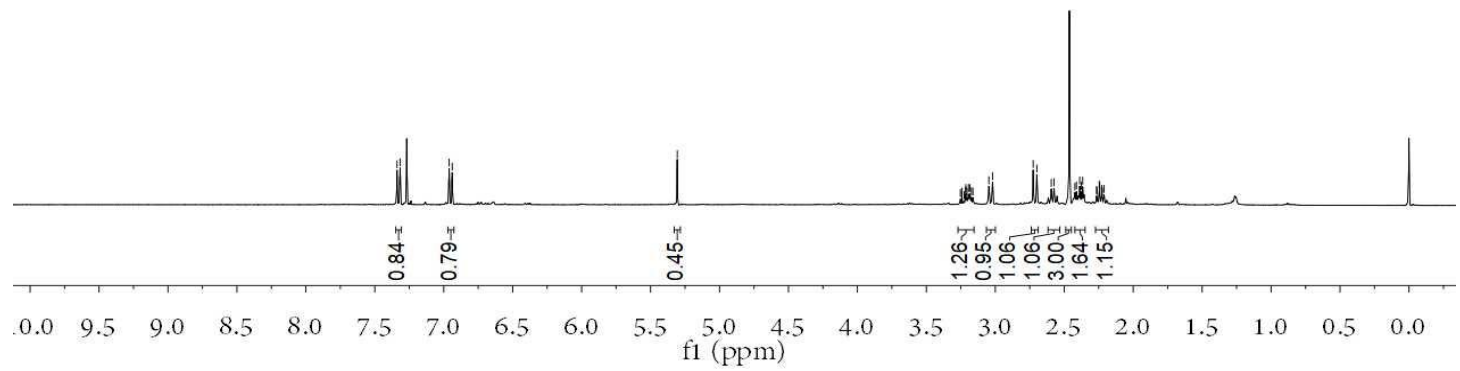

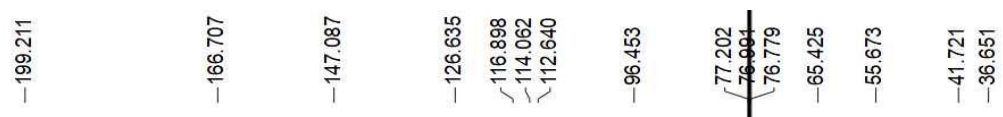

$\begin{array}{lllllllllllllllllllllllll}220 & 210 & 200 & 190 & 180 & 170 & 160 & 150 & 140 & 130 & 120 & 110 & 100 & 90 & 80 & 70 & 60 & 50 & 40 & 30 & 20 & 10 & 0 & -10\end{array}$

Figure S23. ${ }^{1} \mathrm{H}(400 \mathrm{M})$ and ${ }^{13} \mathrm{C}\left\{{ }^{1} \mathrm{H}\right\}(151 \mathrm{M})$ NMR spectra of $4 \mathrm{~b}$ in $\mathrm{CDCl}_{3}$ 
<smiles>Cc1c(O)cc2c(c1C)N(C)C(=O)CC(=O)CC2</smiles>



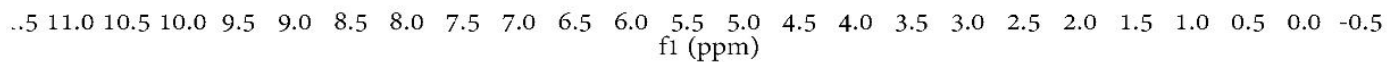

Figure S24. ${ }^{1} \mathrm{H}(600 \mathrm{M})$ and ${ }^{13} \mathrm{C}\left\{{ }^{1} \mathrm{H}\right\}(151 \mathrm{M}) \mathrm{NMR}$ spectra of $4 \mathrm{c}$ in $\mathrm{CDCl}_{3}$ 
<smiles>CCN1C(=O)CC(=O)CCc2cc(O)c(C)c(C)c21</smiles>

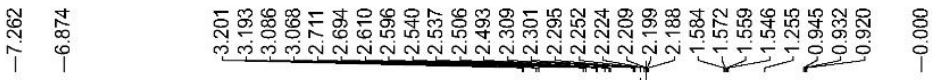
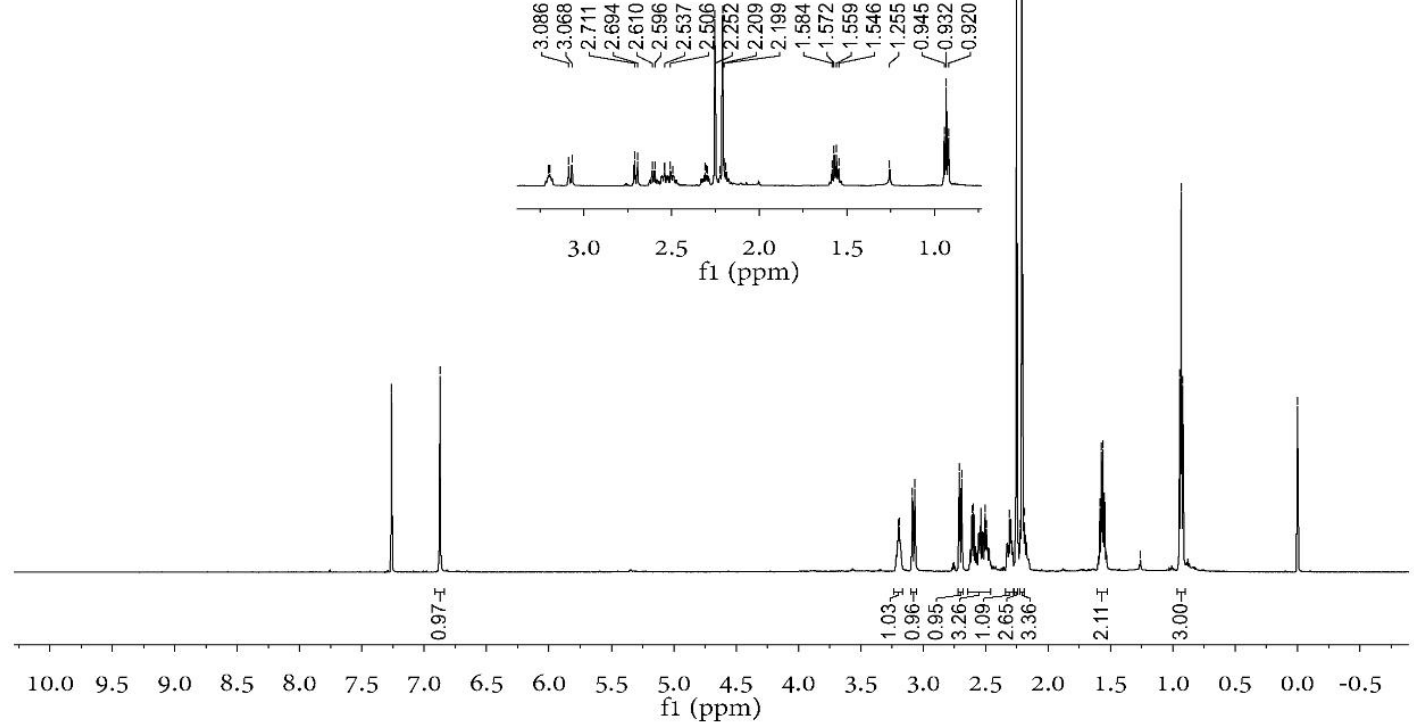

$\begin{array}{llllllllllllllllllllllllll}230 & 220 & 210 & 200 & 190 & 180 & 170 & 160 & 150 & 140 & 130 & 120 & 110 & 100 & 90 & 80 & 70 & 60 & 50 & 40 & 30 & 20 & 10 & 0 & -10\end{array}$ fl $(\mathrm{ppm})$

Figure S25. ${ }^{1} \mathrm{H}(600 \mathrm{M})$ and ${ }^{13} \mathrm{C}\left\{{ }^{1} \mathrm{H}\right\}(151 \mathrm{M}) \mathrm{NMR}$ spectra of $4 \mathrm{~d}$ in $\mathrm{CDCl}_{3}$ 
<smiles>Cc1c(O)cc2c3c1CCC(=O)CC(=O)N3P(C)C2</smiles>

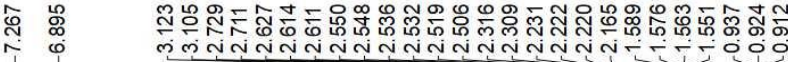
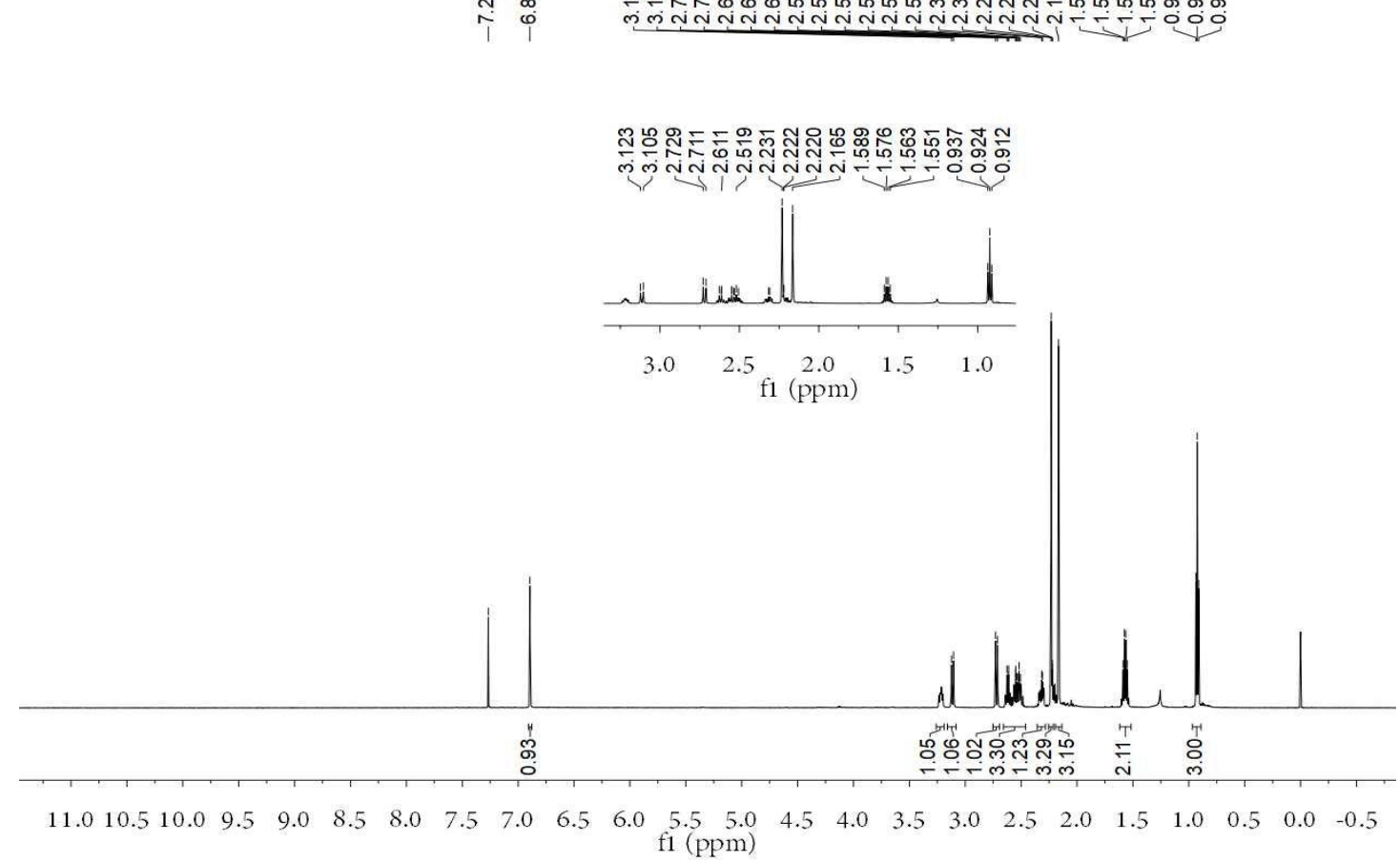

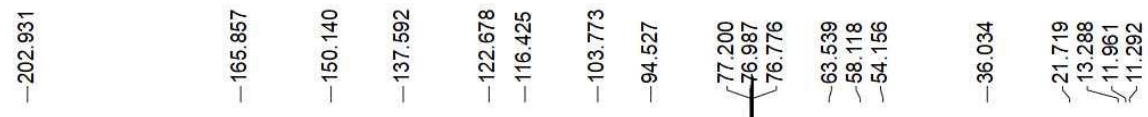

$\begin{array}{lllllllllllllllllllllllllll}230 & 220 & 210 & 200 & 190 & 180 & 170 & 160 & 150 & 140 & 130 & 120 & 110 & 100 & 90 & 80 & 70 & 60 & 50 & 40 & 30 & 20 & 10 & 0 & -10\end{array}$ f1 (ppm)

Figure S26. ${ }^{1} \mathrm{H}(600 \mathrm{M})$ and ${ }^{13} \mathrm{C}\left\{{ }^{1} \mathrm{H}\right\}(151 \mathrm{M})$ NMR spectra of $4 \mathrm{e}$ in $\mathrm{CDCl}_{3}$ 
<smiles>Cc1c(O)cc2c(c1C)N(Br)C(=O)CC(=O)C2</smiles>

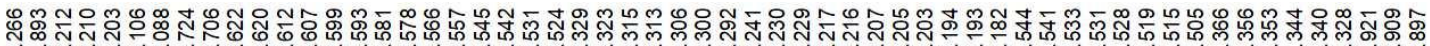

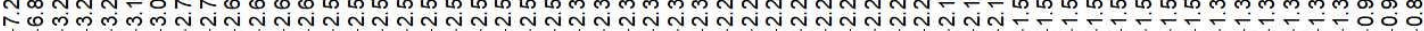
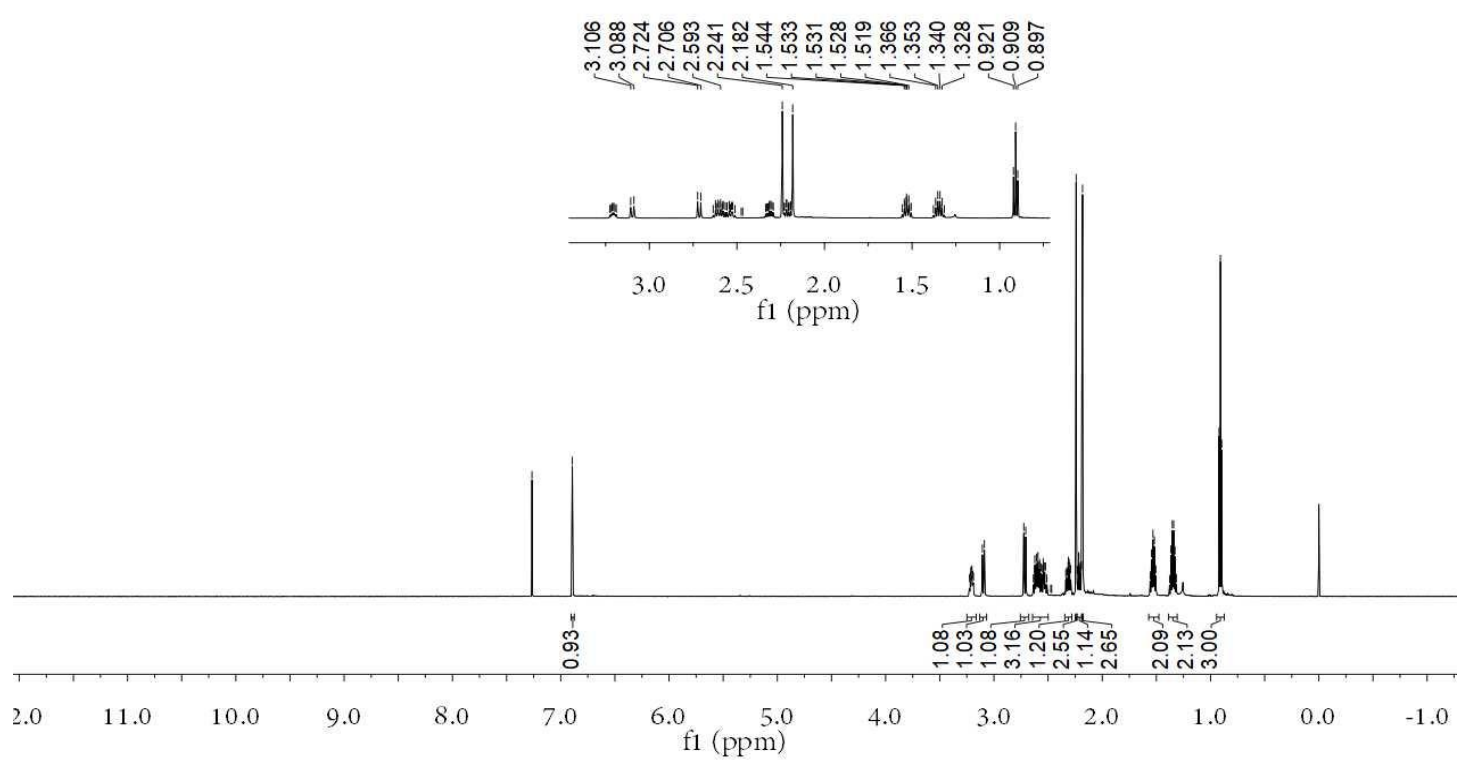

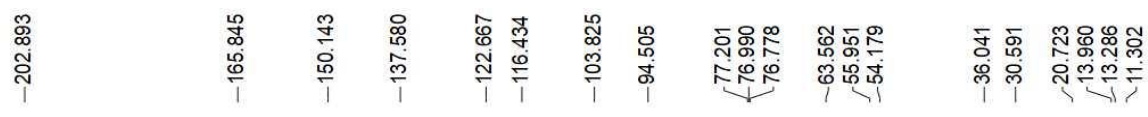

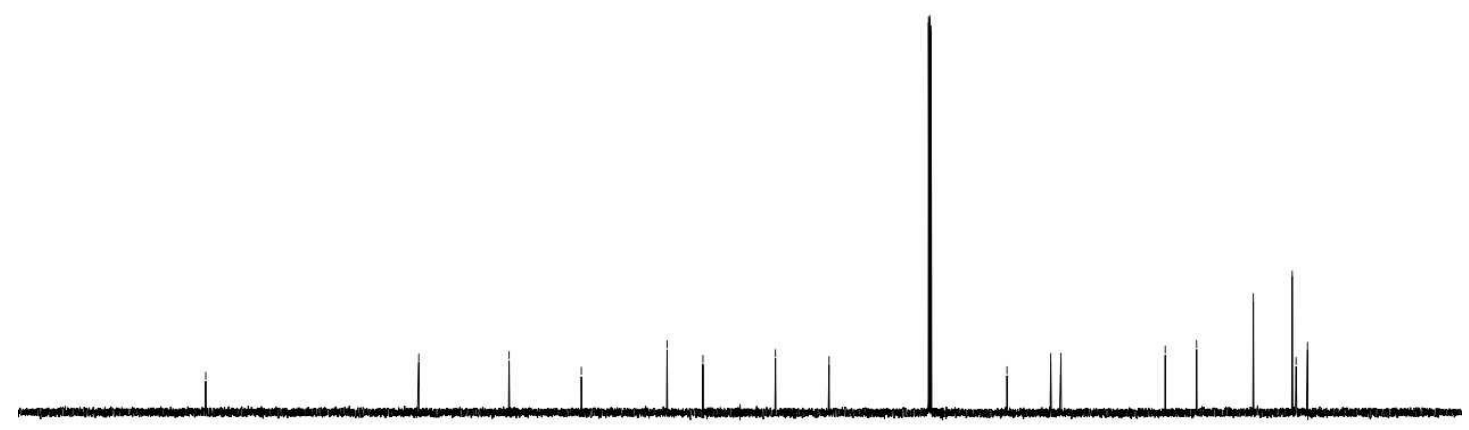

$\begin{array}{lllllllllllllllllllllllll}230 & 220 & 210 & 200 & 190 & 180 & 170 & 160 & 150 & 140 & 130 & 120 & 110 & 100 & 90 & 80 & 70 & 60 & 50 & 40 & 30 & 20 & 10 & 0 & -10\end{array}$ f1 (ppm)

Figure S27. ${ }^{1} \mathrm{H}(600 \mathrm{M})$ and ${ }^{13} \mathrm{C}\left\{{ }^{1} \mathrm{H}\right\}(151 \mathrm{M}) \mathrm{NMR}$ spectra of $4 \mathrm{f}$ in $\mathrm{CDCl}_{3}$ 


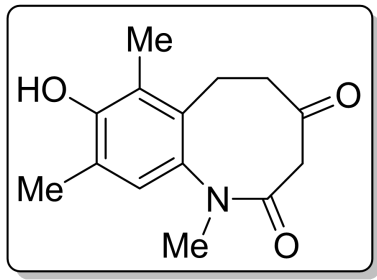





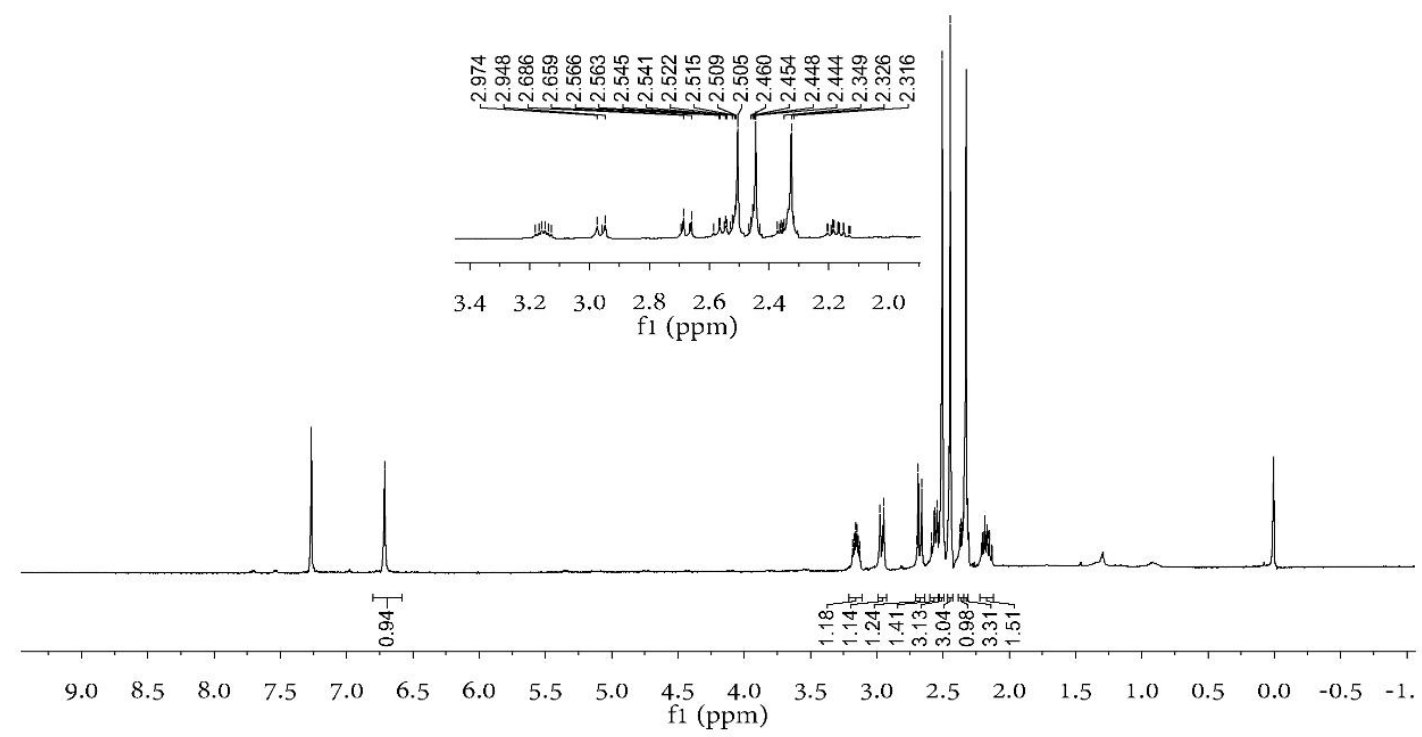

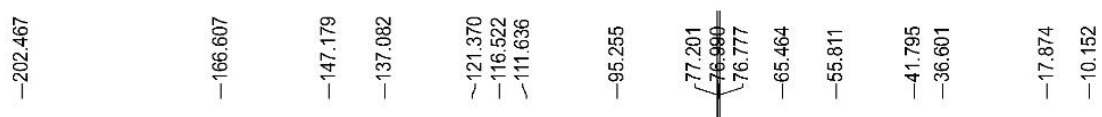

$\begin{array}{llllllllllllllllllllllllll}230 & 220 & 210 & 200 & 190 & 180 & 170 & 160 & 150 & 140 & 130 & 120 & 110 & 100 & 90 & 80 & 70 & 60 & 50 & 40 & 30 & 20 & 10 & 0 & -10\end{array}$

Figure S28. ${ }^{1} \mathrm{H}(400 \mathrm{M})$ and ${ }^{13} \mathrm{C}\left\{{ }^{1} \mathrm{H}\right\}(151 \mathrm{M})$ NMR spectra of $4 \mathrm{~g}$ in $\mathrm{CDCl}_{3}$ 
<smiles>Cc1cc2c(c(C)c1O)CCC(=O)CC2</smiles>

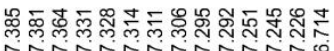

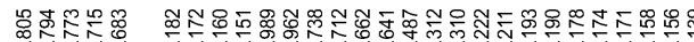



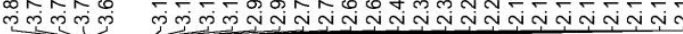

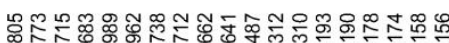
imm

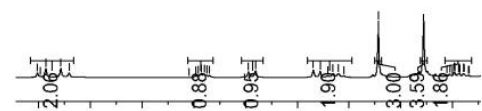

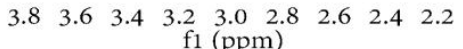

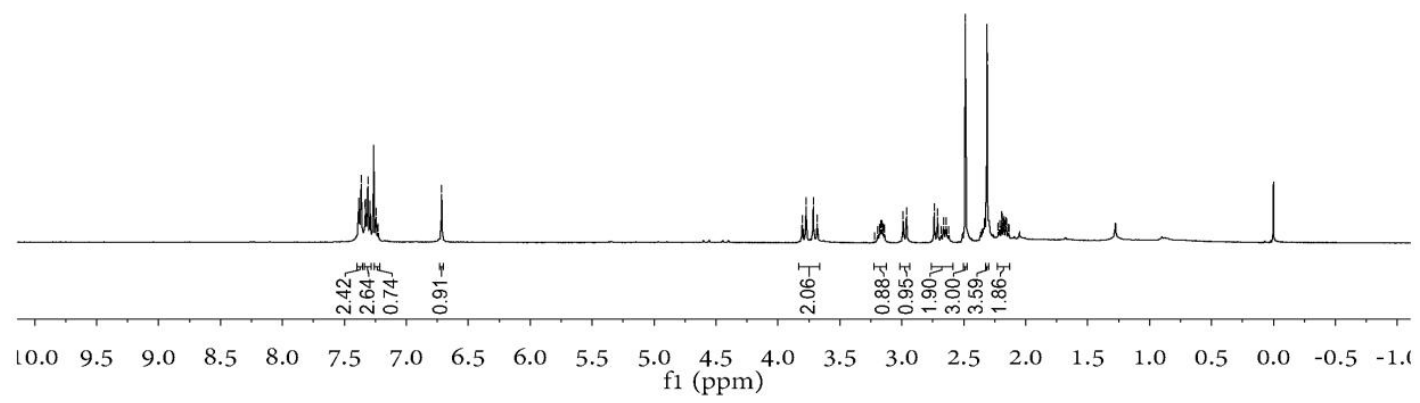

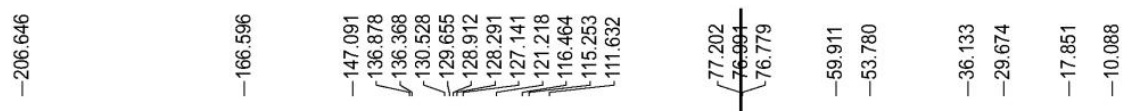

$\begin{array}{lllllllllllllllllllllllll}230 & 220 & 210 & 200 & 190 & 180 & 170 & 160 & 150 & 140 & 130 & 120 & 110 & 100 & 90 & 80 & 70 & 60 & 50 & 40 & 30 & 20 & 10 & 0 & -10\end{array}$ f1 (ppm)

Figure S29. ${ }^{1} \mathrm{H}(400 \mathrm{M})$ and ${ }^{13} \mathrm{C}\left\{{ }^{1} \mathrm{H}\right\}(151 \mathrm{M})$ NMR spectra of $4 \mathrm{~h}$ in $\mathrm{CDCl}_{3}$ 
<smiles>CN1C(=O)CCCc2c(Br)c(O)cc(Br)c21</smiles>



$\begin{array}{lllllllllllllllllllllllll}1.5 & 11.0 & 10.5 & 10.0 & 9.5 & 9.0 & 8.5 & 8.0 & 7.5 & 7.0 & 6.5 & 6.0 & 5.5 & 5.0 & 4.5 & 4.0 & 3.5 & 3.0 & 2.5 & 2.0 & 1.5 & 1.0 & 0.5 & 0.0 & -0.5\end{array}$

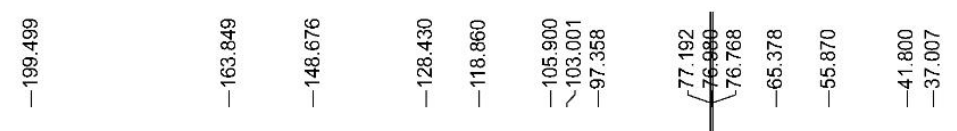

$\begin{array}{llllllllllllllllllllllllll}230 & 220 & 210 & 200 & 190 & 180 & 170 & 160 & 150 & 140 & 130 & 120 & 110 & 100 & 90 & 80 & 70 & 60 & 50 & 40 & 30 & 20 & 10 & 0 & -10\end{array}$ f1 (ppm)

Figure S30. ${ }^{1} \mathrm{H}(600 \mathrm{M})$ and ${ }^{13} \mathrm{C}\left\{{ }^{1} \mathrm{H}\right\}(151 \mathrm{M})$ NMR spectra of $4 \mathrm{i}$ in $\mathrm{CDCl}_{3}$ 
<smiles>CCN1C(=O)CCCc2c(Br)c(O)cc(Br)c21</smiles>

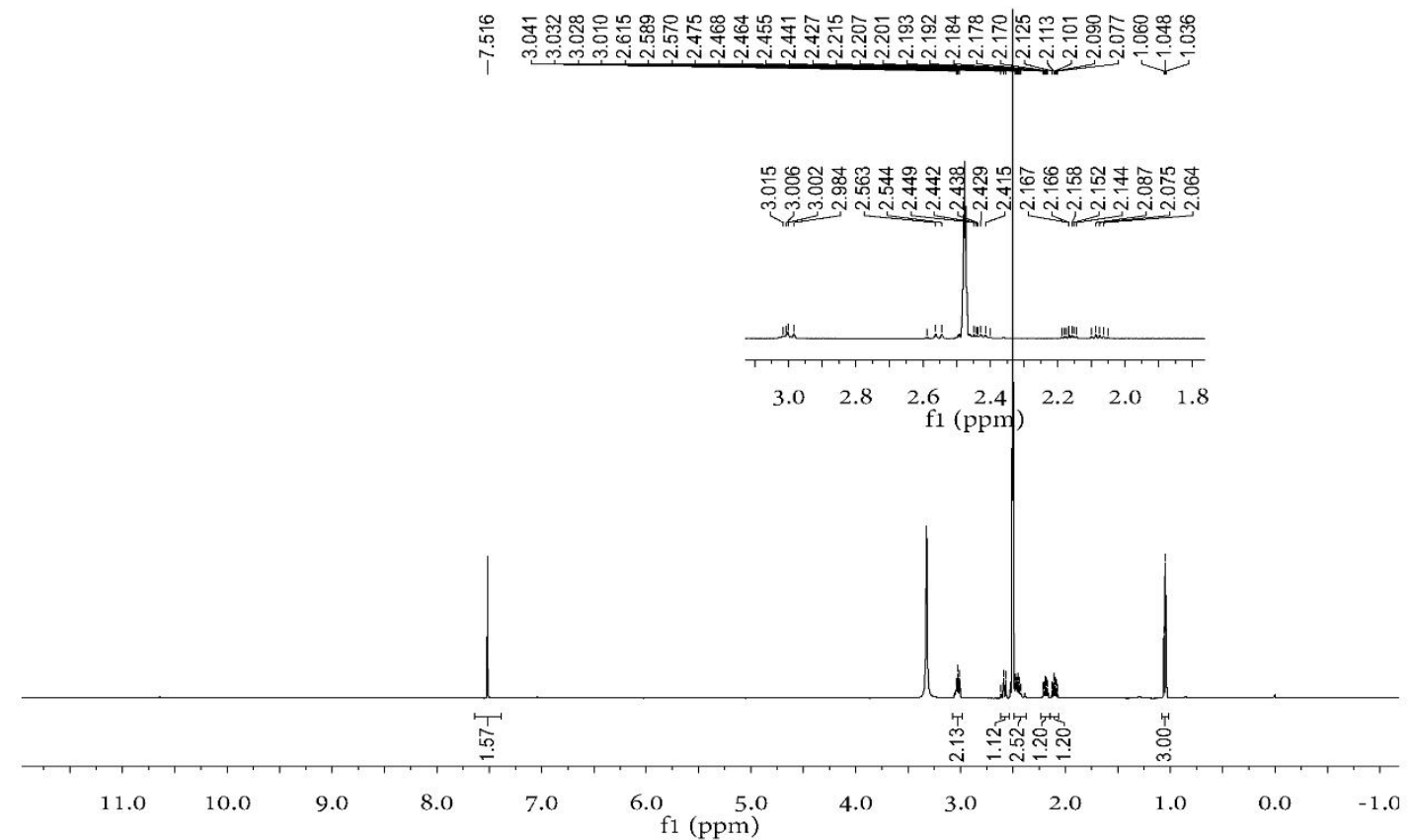

\begin{tabular}{|c|c|c|c|c|c|}
\hline 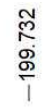 &  &  & $\underset{\stackrel{N}{\stackrel{N}{*}}}{\stackrel{1}{\sim}}$ & & 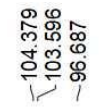 \\
\hline
\end{tabular}

Figure S31. ${ }^{1} \mathrm{H}(600 \mathrm{M})$ and ${ }^{13} \mathrm{C}\left\{{ }^{1} \mathrm{H}\right\}(151 \mathrm{M})$ NMR spectra of $4 \mathrm{j}$ in DMSO-d 6 
<smiles>O=C1CCc2c(Br)c(O)cc(Br)c2N(P)C(=O)C1</smiles>

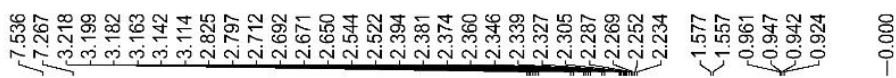


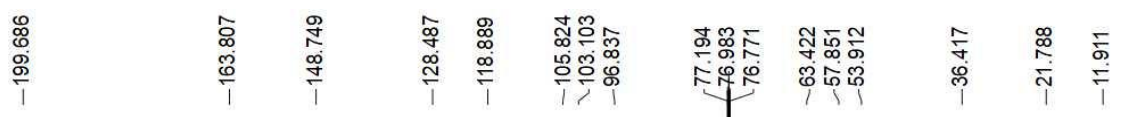

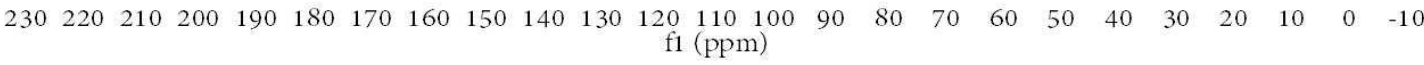

Figure S32. ${ }^{1} \mathrm{H}(400 \mathrm{M})$ and ${ }^{13} \mathrm{C}\left\{{ }^{1} \mathrm{H}\right\}(151 \mathrm{M}) \mathrm{NMR}$ spectra of $4 \mathrm{k}$ in $\mathrm{CDCl}_{3}$ 
<smiles>O=C1CCc2c(Br)c(O)cc(Br)c2N(Br)C(=O)C1</smiles>

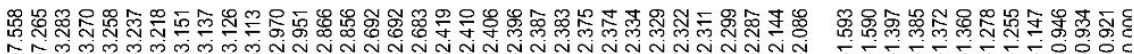
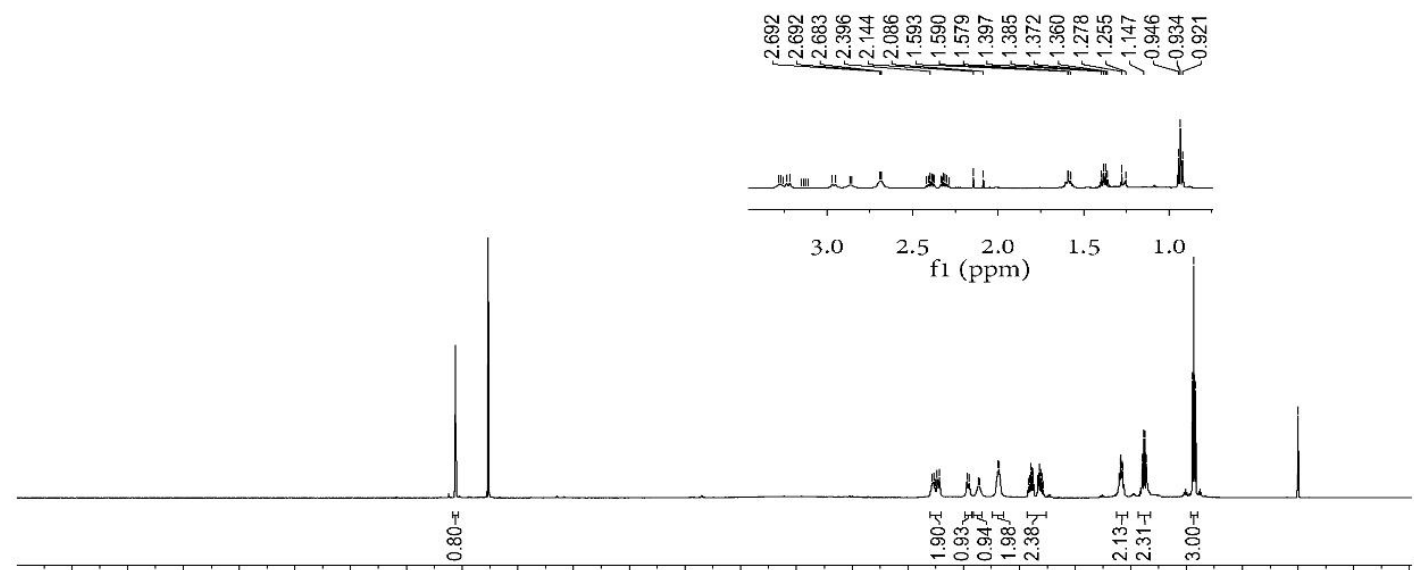

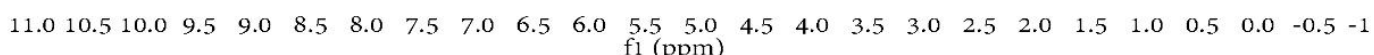

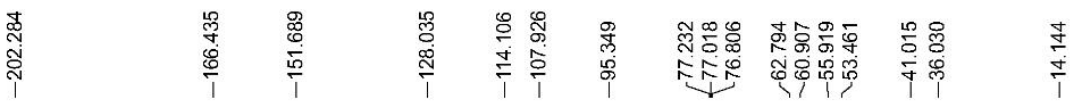

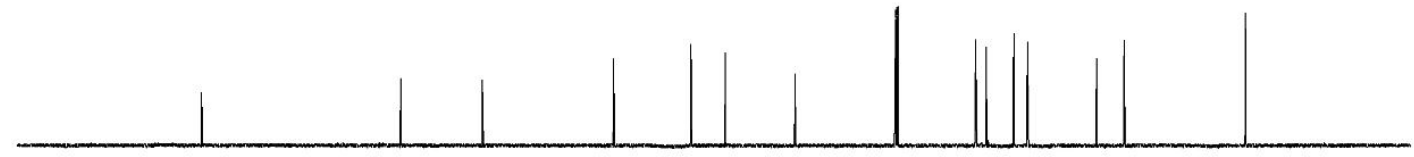

$\begin{array}{llllllllllllllllllllllllll}230 & 220 & 210 & 200 & 190 & 180 & 170 & 160 & 150 & 140 & 130 & 120 & 110 & 100 & 90 & 80 & 70 & 60 & 50 & 40 & 30 & 20 & 10 & 0 & -10\end{array}$ f1 (ppm)

Figure S33. ${ }^{1} \mathrm{H}(600 \mathrm{M})$ and ${ }^{13} \mathrm{C}\left\{{ }^{1} \mathrm{H}\right\}(151 \mathrm{M}) \mathrm{NMR}$ spectra of 41 in $\mathrm{CDCl}_{3}$ 
7,10-Dibromo-1-cyclopropyl-8-hydroxy-5,6-dihydrobenzo[b]azocine-2,4(1H, 3H)-dione $(4 \mathrm{~m})$<smiles>O=C1CCc2c(Br)c(O)cc(Br)c2N(C2CC2)C(=O)C1</smiles>

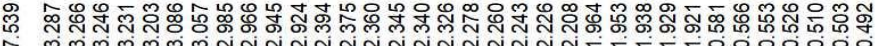

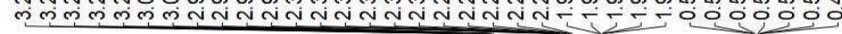



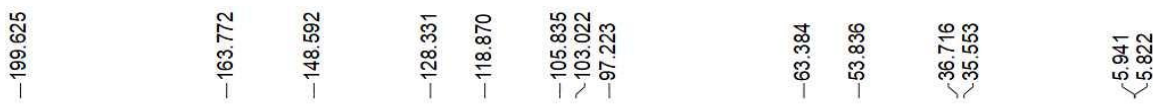



$\begin{array}{lllllllllllllllllllllllll}230 & 220 & 210 & 200 & 190 & 180 & 170 & 160 & 150 & 140 & 130 & 120 & 110 & 100 & 90 & 80 & 70 & 60 & 50 & 40 & 30 & 20 & 10 & 0 & -10\end{array}$ f1 (ppm)

Figure S34. ${ }^{1} \mathrm{H}(400 \mathrm{M})$ and ${ }^{13} \mathrm{C}\left\{{ }^{1} \mathrm{H}\right\}(151 \mathrm{M}) \mathrm{NMR}$ spectra of $4 \mathrm{~m}$ in $\mathrm{CDCl}_{3}$ 

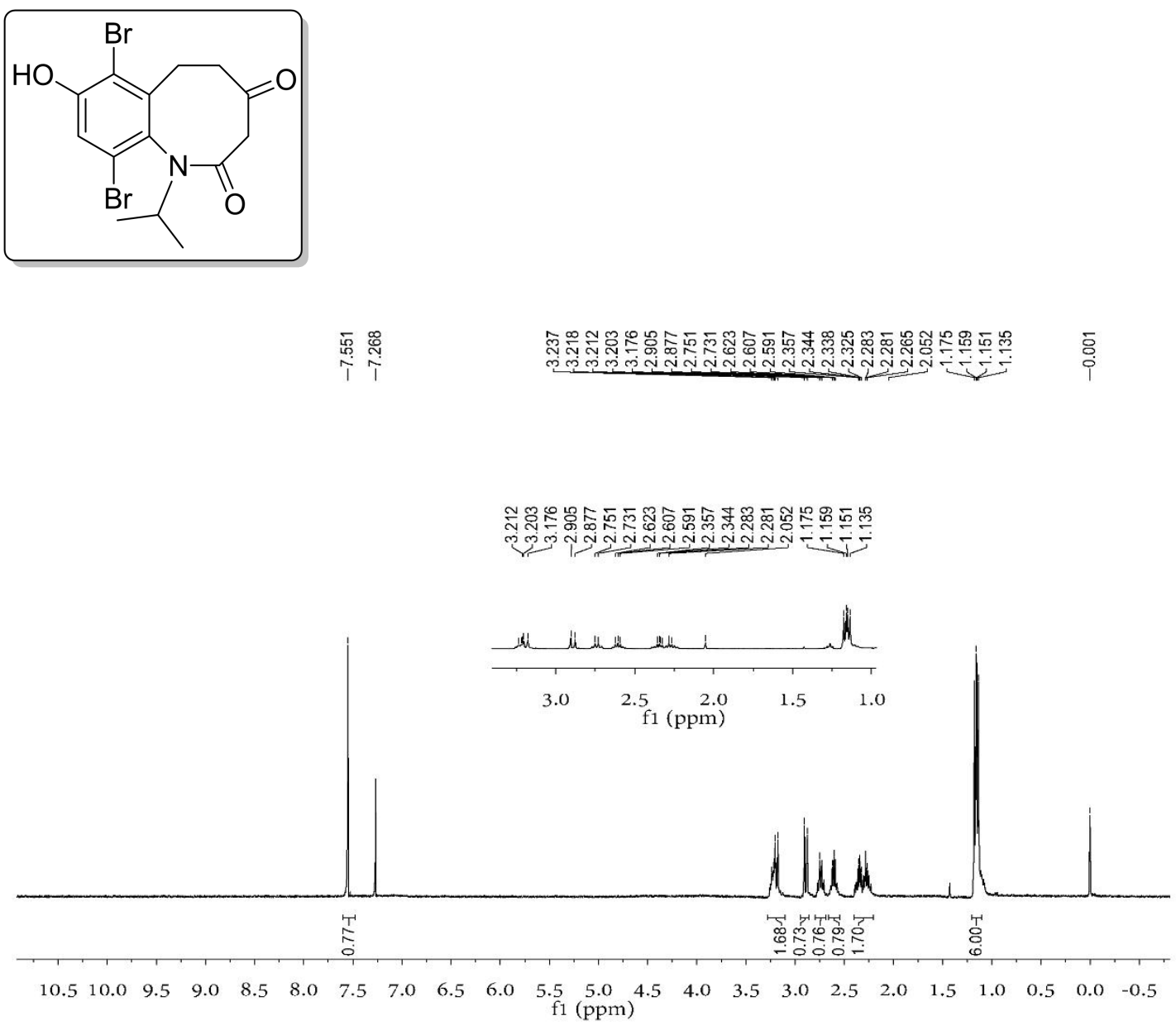

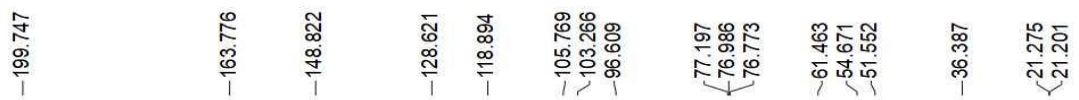

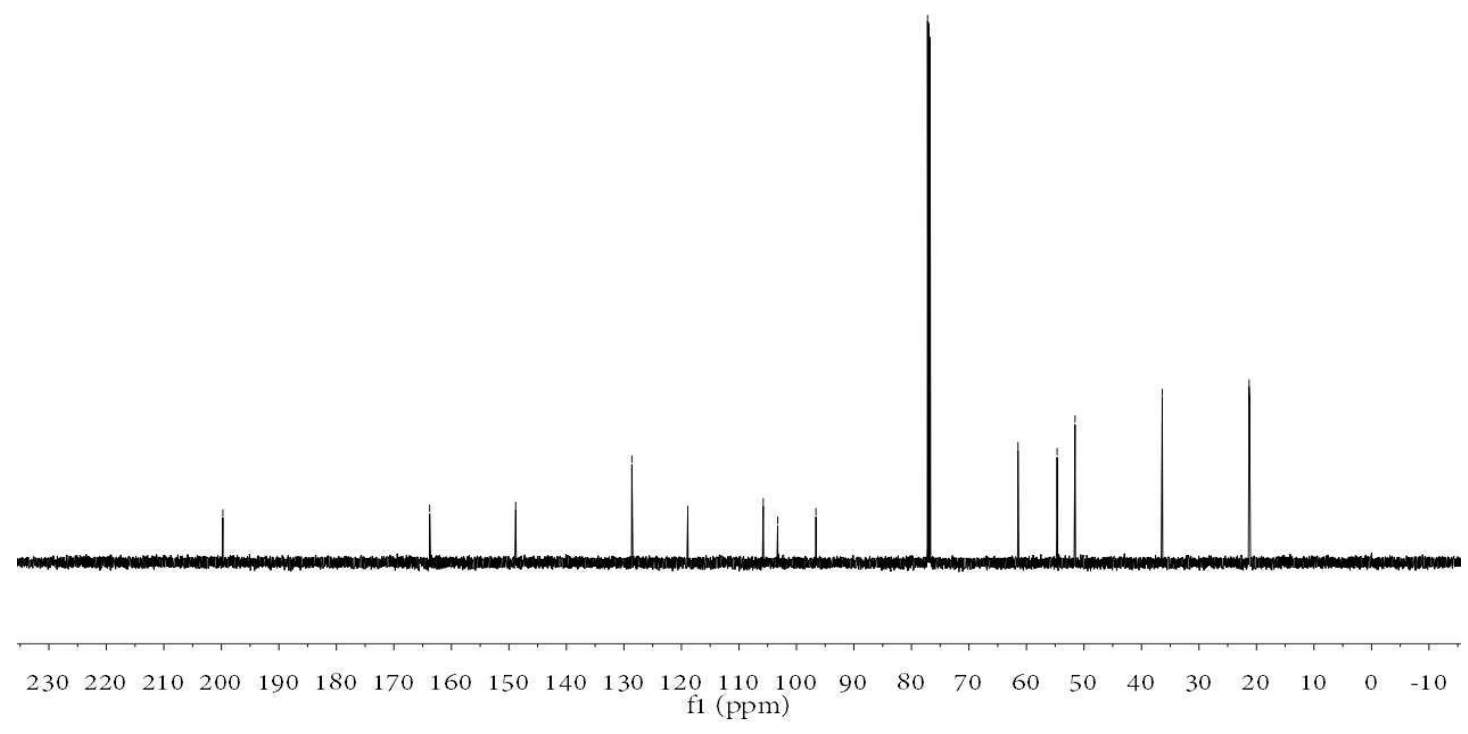

Figure S35. ${ }^{1} \mathrm{H}(400 \mathrm{M})$ and ${ }^{13} \mathrm{C}\left\{{ }^{1} \mathrm{H}\right\}(151 \mathrm{M}) \mathrm{NMR}$ spectra of $4 \mathrm{n}$ in $\mathrm{CDCl}_{3}$ 
7,10-Dibromo-8-hydroxy-1,3-dimethyl-5,6-dihydrobenzo[b]azocine-2,4(1H,3H)-dione (4p)<smiles>CC1C(=O)CCc2c(Br)c(O)cc(Br)c2N1C</smiles>

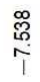

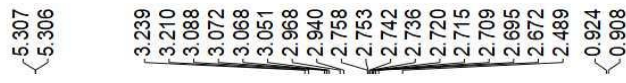

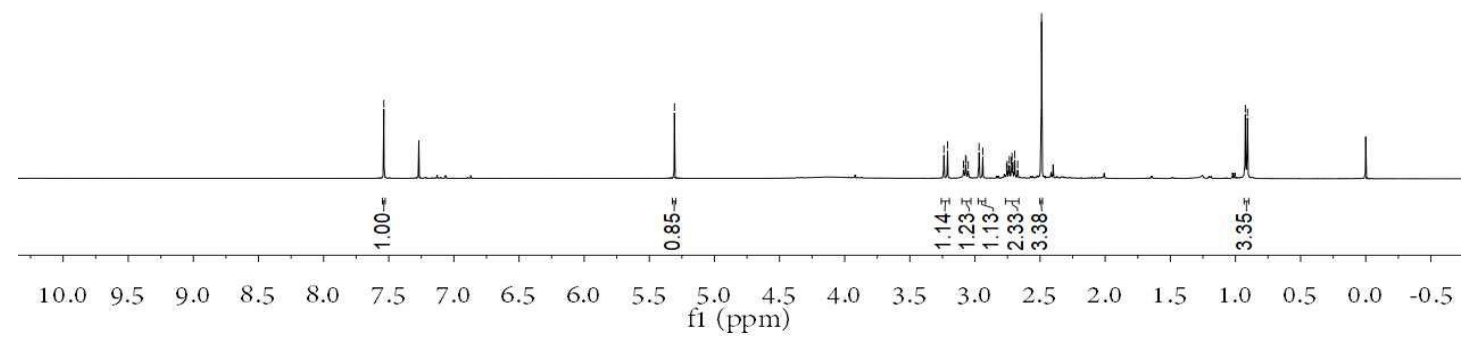

\begin{tabular}{|c|c|c|c|c|c|c|c|c|c|}
\hline 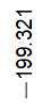 & 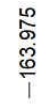 & $\begin{array}{l}\hat{N} \\
\infty \\
\infty \\
0 \\
+ \\
1\end{array}$ & $\begin{array}{l}\dddot{0} \\
\stackrel{0}{0} \\
\stackrel{\infty}{0} \\
\stackrel{1}{1}\end{array}$ & $\begin{array}{l}80 \\
6 \\
\circ \\
\end{array}$ &  & 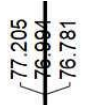 & 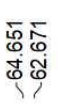 & 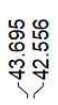 & 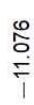 \\
\hline
\end{tabular}

$\begin{array}{lllllllllllllllllllllllll}230 & 220 & 210 & 200 & 190 & 180 & 170 & 160 & 150 & 140 & 130 & 120 & 110 & 100 & 90 & 80 & 70 & 60 & 50 & 40 & 30 & 20 & 10 & 0 & -10\end{array}$

Figure S36. ${ }^{1} \mathrm{H}(400 \mathrm{M})$ and ${ }^{13} \mathrm{C}\left\{{ }^{1} \mathrm{H}\right\}(151 \mathrm{M})$ NMR spectra of $4 \mathrm{p}$ in $\mathrm{CDCl}_{3}$ 